\title{
Charting the Parameter Space of the 21-cm Power Spectrum
}

\author{
Aviad Cohen ${ }^{1 \star}$, Anastasia Fialkov², Rennan Barkana ${ }^{1,3,4,5}$ \\ 1 Raymond and Beverly Sackler School of Physics and Astronomy, Tel Aviv University, Tel Aviv 69978, Israel \\ 2 Department of Astronomy, Harvard University, 60 Garden Street, MS51, Cambridge, MA, 02138 U.S.A. \\ 3 Sorbonne Universités, Institut Lagrange de Paris (ILP), Institut d'Astrophysique de Paris, UPMC Univ Paris 06/CNRS \\ 4 Department of Astrophysics, University of Oxford, Denys Wilkinson Building, Keble Road, Oxford OX1 3RH, UK \\ 5 Perimeter Institute for Theoretical Physics, 31 Caroline St N., Waterloo, ON N2L 2Y5, Canada
}

8 September 2017

\begin{abstract}
The high-redshift 21-cm signal of neutral hydrogen is expected to be observed within the next decade and will reveal epochs of cosmic evolution that have been previously inaccessible. Due to the lack of observations, many of the astrophysical processes that took place at early times are poorly constrained. In recent work we explored the astrophysical parameter space and the resulting large variety of possible global (sky-averaged) $21-\mathrm{cm}$ signals. Here we extend our analysis to the fluctuations in the 21-cm signal, accounting for those introduced by density and velocity, Ly $\alpha$ radiation, $\mathrm{X}$-ray heating, and ionization. While the radiation sources are usually highlighted, we find that in many cases the density fluctuations play a significant role at intermediate redshifts. Using both the power spectrum and its slope, we show that properties of high-redshift sources can be extracted from the observable features of the fluctuation pattern. For instance, the peak amplitude of ionization fluctuations can be used to estimate whether heating occurred early or late and, in the early case, to also deduce the cosmic mean ionized fraction at that time. The slope of the power spectrum has a more universal redshift evolution than the power spectrum itself and can thus be used more easily as a tracer of high-redshift astrophysics. Its peaks can be used, for example, to estimate the redshift of the Ly $\alpha$ coupling transition and the redshift of the heating transition (and the mean gas temperature at that time). We also show that a tight correlation is predicted between features of the power spectrum and of the global signal, potentially yielding important consistency checks.
\end{abstract}

Key words: galaxies: formation - galaxies: high redshift - intergalactic medium cosmology: theory

\section{INTRODUCTION}

The most promising probe of the early universe and the epoch of primordial star formation (cosmic dawn) is the redshifted spectral line of atomic hydrogen, which has a restframe wavelength of $21 \mathrm{~cm}$. This signal is expected to be produced prior to complete reionization by abundant neutral hydrogen in the intergalactic medium (IGM) and should allow us to explore cosmic history down to $z \sim 6$. Because the signal depends both on the cosmological model and on astrophysics, it contains abundant information about the Universe at early times (e.g., Furlanetto et al. 2006; Barkana 2016).

Observational efforts to date have resulted in lim-

* E-mail: aviadc11@gmail.com its on both the global 21-cm signal and the power spectrum of 21-cm fluctuations. Experiments that are currently taking (or analyzing) data to detect the power spectrum from the epoch of reionization (EoR) include the Low Frequency Array (LOFAR, Patil et al. 2017), the Precision Array to Probe the Epoch of Reionization (PAPER, Parsons et al. 2014; Jacobs et al. 2015; Ali et al. 2015), the Murchison Wide-field Array (MWA, Bowman et al. 2013; Beardslev et al. 2016), and the Hydrogen Epoch of Reionization Array (HERA, Pober et al. 2014; DeBoer et al. 2016); meanwhile, an effort is being made to measure the global signal at both the low-redshift (EoR) and high-redshift (cosmic dawn) regimes, by EDGES (the Experiment to Detect the Global EoR Signature, Bowman \& Rogers 2010; Monsalve et al. 2017), SARAS (the Shaped Antenna measurement of the background RAdio Spectrum, Singh et al.

(C) 2017 RAS 
2017), and LEDA (Large-Aperture Experiment to Detect the Dark Ages, Bernardi et al. 2016). Planned experiments (or under construction) include the New Extension in Nancay Upgrading LOFAR (NenuFAR, Zarka et al. 2012), the Square Kilometer Array (SKA, Koopmans et al. 2015), and the Dark Ages Radio Explorer (DARE, Burns et al. 2015); these instruments will probe both the global signal and its power spectrum over a wide range of epochs.

Observing the 21-cm signal will shed light on the astrophysical processes that shaped the young Universe. One of the most important questions that can be answered by detecting this signal is how some of the first stars came to be. The abundance and spatial distribution of high redshift galaxies determines the pattern of the radiative backgrounds produced by sources of light, and thus has a strong effect on the fluctuations in the 21-cm signal. A major parameter in this is the minimum mass of star forming halos, $M_{\min }$, which is usually set by the requirement of efficient gas cooling. Because they are formed in highly over-dense regions, massive halos are rarer and more highly clustered than lighter ones (Barkana \& Loeb 2004), and imprint stronger fluctuations in the signal. Theoretical work shows that star formation in a dark matter halo becomes possible only if the halo is massive enough to radiatively cool the infalling gas (e.g., Tegmark et al. 1997). For instance, molecular hydrogen, $\mathrm{H}_{2}$, one of the available building blocks in the pristine environment at high redshifts, has to be gravitationally accelerated and shock-heated to temperatures higher than $\sim 300 \mathrm{~K}$ in order to initiate radiative cooling, which leads to star formation in halos above $M_{\min } \sim 10^{5} \mathrm{M}_{\odot}$ (e.g., Tegmark et al. 1997; Bromm et al. 2002; Yoshida et al. 2003). If molecular hydrogen is unavailable, stars will form via cooling of atomic hydrogen in more massive halos, above $M_{\min } \sim 10^{7} \mathrm{M}_{\odot}$ (e.g., Barkana \& Loeb 2001).

Formation of stars through cooling of $\mathrm{H}_{2}$ is sensitive to various feedback processes. In particular, radiation emitted by stars includes photons in the Lyman-Werner (LW) band (11.2-13.6 eV), which dissociate hydrogen molecules (Haiman et al. 1997) thus boosting the minimal mass of star forming halos (Haiman et al. 2000; Machacek et al. 2001; Wise \& Abel 2007; O'Shea \& Norman 2008). The efficiency of this feedback mechanism, and thus the transition point of star formation from molecular cooling to atomic cooling, are highly uncertain (Visbal et al. 2014; Schauer et al. 2015). In addition to the LW feedback, star formation in small halos is sensitive to other factors, such as the relative streaming velocity between dark matter and gas, which suppresses star formation in a spatially inhomogeneous way in halos below $\sim$ $10^{6} \mathrm{M}_{\odot}$ Tseliakhovich \& Hirata 2010; Dalal, Pen, \& Seliak 2010; Tseliakhovich et al. 2011; Visbal et al. 2012; Fialkov 2014). Another possible way to quench star formation in small halos is via supernova explosions that can expel gas from light halos, raising $M_{\min }$ well above the atomic cooling threshold (e.g., Wvithe \& Loeb 2013). On the other hand, supernova explosions can also revive star formation in light halos by enriching gas with metals. Because metal-rich gas can allow a lower $M_{\min }$ than even the $\mathrm{H}_{2}$ cooling channel, small halos may contribute to star formation at high redshifts via metal-line cooling despite the effect of the LW feedback. Current numerical simulations suggest that star formation is likely to be inefficient in small halos, but whether metal cooling contributes significantly to high-redshift star formation is still uncertain (e.g., Jeon et al. 2014; Wise et al. 2014; O'Shea et al. 2015; Cohen et al. 2016a). Finally, when the gas in the IGM is photoheated above $10^{4} \mathrm{~K}$ by ionizing photons, photoheating feedback becomes efficient and gas stops accreting onto halos below $10^{8}-10^{9} \mathrm{M}_{\odot}$ (e.g., Rees 1986; Weinberg et al. 1997; Navarro \& Steinmetz 2000; Sobacchi \& Mesinger 2013; Cohen et al. 2016a). However, this process becomes significant only at relatively low redshifts, during the advanced stages of reionization. Future 21-cm measurements will be able to constrain the cooling channel and the efficiency of primordial star formation.

Stars and their remnants produce radiative backgrounds that strongly affect the environment. For instance, the temperature of the IGM rises due to the $\mathrm{X}$-ray radiation produced by the first heating sources. Even fixing the total energy emitted in X-rays, different spectral energy distributions (SEDs) can lead to completely different heating histories and, thus, predicted 21-cm signals (Fialkov et al. 2014a; Pacucci et al. 2014; Fialkov \& Barkana 2014). Because the mean free path is larger for high-energy photons, hard photons travel further away from the source before depositing their energy into the IGM. This leads to a delayed, weaker (due to redshift losses), and more spatially uniform heating of the universe in the case of sources with a hard SED. The temperature of the gas directly affects the $21-\mathrm{cm}$ intensity. As a result, X-ray sources imprint their signature in the signal from neutral hydrogen. By detecting the characteristic signature, the nature and distribution of the first X-ray sources can be studied. At present, the high-redshift X-ray population is poorly constrained; however, available observations yield upper and lower limits on the X-ray luminosity of the sources (Fialkov et al. 2017). Upper limits come from the unresolved soft X-ray background (Dijkstra et al. 2012; Fialkov et al. 2017), while lower limits can be extracted from the upper limits on the $21-\mathrm{cm}$ power spectrum Ali et al. 2015; Pober et al. 2015).

There are several candidate X-ray sources discussed in the literature, and at present it is unclear which one dominates at high redshift. High-mass X-ray binaries (XRBs) are currently the most plausible dominant source (Mirabel et al. 2011). This possibility is supported by a detailed population synthesis simulation that indicated that XRBs dominate over the contribution of quasars at $z \gtrsim 6-8$ (Fragos et al. 2013). Although the majority of X-ray photons emitted by XRBs are hard, with the X-ray SED peaking around $1-3$ $\mathrm{keV}$, the SED used in previous models has often been a soft power law (e.g., Furlanetto 2006), which might be more appropriate for describing emission by gas heated by supernova explosions within galaxies. The third possible source of Xray radiation is a population of mini-quasars (MQ), central black holes in early star-forming halos (Madau et al. 2004). The properties of these objects, including their contribution to heating and reionization, are highly uncertain, since their masses and the characteristics of their host galaxies are very different from those of the central black holes observed today in much more massive halos. Because it takes time for a supermassive black hole to grow, mini-quasars tend to be significant only in relatively massive halos and, therefore, in our models their heating becomes important at lower redshifts than the other two options. The bolometric luminosity of Xray sources, $L_{X}$, is another free parameter. Both population synthesis simulations (Fragos et al. 2013) and observations 
(Brorby et al. 2016) suggest that in the case of XRBs, $L_{X}$ is larger in metal-poor high-redshift galaxies compared to their metal-rich low-redshift counterparts. The same studies also indicate that the luminosity is proportional to the star formation rate (SFR), allowing us to define a $L_{X}$-SFR relation. However, the exact relation between $L_{X}$ and the SFR depends on the nature of the sources, and it is highly uncertain, especially at high redshifts where there are few observational constraints.

In addition to X-ray radiation, the first luminous objects emitted ultraviolet (UV) radiation, which ionized the neutral hydrogen in the IGM. Thanks to the effect of ionized gas on the CMB, the value of this parameter is relatively well constrained (compared to the other parameters that we consider), with a total scattering optical depth of $\tau=0.055 \pm 0.009$ (Planck Collaboration et al. 2016a). The main sources of ionizing radiation are believed to be stars; however, reionization by quasars may also be possible (Madau \& Haardt 2015).

In previous work (Cohen et al. 2016b), we explored the space of astrophysical parameters, varying the parameters that play crucial roles in driving the $21-\mathrm{cm}$ signal, namely: the minimal mass of star-forming halos and the star formation efficiency (which reflect the dominant cooling channel and the efficiency of internal feedback), the parameters of X-ray heating (the SED and the bolometric luminosity of $\mathrm{X}$-ray sources), and the total reionization optical depth and maximum mean free path of ionizing photons. In that work, we used our simulations to predict the global 21-cm signal (i.e., the mean spectrum over the relevant frequency range), for 193 different combinations of the astrophysical parameters. We showed that the expected signal fills a large parameter space, but with a fixed general shape of the global 21-cm signal. Using the 193 models we identified relations between features of the spectrum and the astrophysical parameters. Since we showed these relations to hold over a very wide range of possible astrophysics parameters, these relations can be used to directly link future measurements of the global signal to astrophysical quantities in a mostly modelindependent way. This approach is novel in that it covers a substantially wider astrophysical parameter space than other work (e.g., Greig \& Mesinger 2015; Hassan et al. 2016; Shimabukuro \& Semelin 2017). For example, such works often focus on the late stages of reionization assuming that cosmic heating has saturated and thus the parameters of the $\mathrm{X}$-ray sources do not matter. They also usually assume that small halos, those below the atomic cooling threshold, do not contribute significant star formation. Such simplifying assumptions are often made because algorithms such as the Monte Carlo Markov Chain analysis tool (Greig \& Mesinger 2015) need to run the simulation many times before they succeed to fill up the required parameter space. However, given the current lack of observational constraints, the space of possibilities is still extremely wide. For example, the relative timing between reionization and the heating era is uncertain. It has been recently recognized that heating occurs late in many scenarios, and the cosmic gas can still be colder than the CMB during the early stages of reionization (Fialkov et al. 2014a; Madau \& Fragos 2016; Mirocha et al. 2016; Cohen et al. 2016b).

This paper is a follow up to Cohen et al. 2016b). Here we use the same compilation of models to map out the space of possible 21-cm power spectra. We explore features of the 21-cm power spectrum and aim to classify the main observable properties of the evolution in redshift of the power spectrum. Another goal of ours is to establish relations between these features and the astrophysical parameters. This paper is organized as follows: In Section 2 we discuss the general properties of the 21-cm power spectrum (Section 2.1), and then outline and discuss the astrophysical parameter space (Section 2.2). In Section 3 we illustrate our predictions in detail for a particular choice of astrophysical parameters, while in Section 4 we generalize our results to the entire parameter space, focusing on correlations between the redshift evolution of the power spectrum (and that of its slope) and the properties of early galaxies. In Section 5 we show that the timing of cosmic milestones (such as the redshift at which the IGM was heated to the temperature of the CMB) can be extracted from the evolution of the slope, as well as from the spectral shape of the global 21-cm signal. In the same Section we also provide consistency relations that could be used to verify experimental results once the measurements of both the global signal and the power spectrum are available. Finally, we summarize and conclude in Section 6 .

\section{SIMULATED 21-CM SIGNAL}

Our goal is to explore the high-redshift astrophysical parameter space and create a mock 21-cm signal for a large number of parameter sets. To this end we use a flexible and fast seminumerical method first introduced by Visbal et al. (2012), inspired by 21cmfast (Mesinger et al. 2011). The framework follows the evolution of the density and velocity fields in time in a large cosmological volume (a $384^{3} \mathrm{Mpc}^{3}$ box) with coarse resolution $(3 \mathrm{Mpc})$, and extensively uses subgrid models to implement physics on smaller scales (Fialkov 2012; Visbal et al. 2012; Fialkov 2013; Fialkov \& Barkana 2014; Fialkov et al. 2014a.b; Cohen et al. 2016a). The star formation rate in each cell, at each redshift and in each halo mass bin is computed using the extended Press-Shechter formalism (Barkana \& Loeb 2004). Assuming population II star formation (Barkana \& Loeb 2005b) and properly accounting for time delay effects, the simulation calculates various radiative backgrounds created by stars and their remnants, including the Ly $\alpha$ background which is needed to source the Wouthuysen-Field (WF) coupling (Wouthuysen 1952; Field 1958), LW radiation responsible for radiative feedback, X-rays that heat the gas, and ionizing UV radiation. This simulation takes into account the effect of relative streaming velocity between dark and baryonic matter (Tseliakhovich \& Hirata 2010; Visbal et al. 2012), and the photoheating feedback (Cohen et al. 2016a). While the code is inspired by $21 \mathrm{cmfast}$ (Mesinger et al. 2011), it goes beyond it with more accurate X-ray heating (including the effect of local reionization), Ly $\alpha$ fluctuations (approximately including the effect of multiple scattering), and photoheating feedback, plus the possibility of having substantial star formation in halos below the atomic cooling threshold, in which case spatially-inhomogeneous processes such as the streaming velocity and LW feedback play a key role (and are included in our 21-cm code but not in others). 


\subsection{The 21-cm power spectrum}

An output of the simulation is the inhomogeneous 21-cm signal calculated for every cell in the redshift range $6-50$. The brightness temperature observed against the CMB is

$T_{\mathrm{b}}=26.8 x_{\mathrm{HI}}\left(\frac{1+z}{10}\right)^{1 / 2}(1+\delta) \frac{x_{\mathrm{tot}}}{1+x_{\mathrm{tot}}}\left[1-\frac{T_{\mathrm{CMB}}}{T_{\text {gas }}}\right] \mathrm{mK}$,

where $x_{\mathrm{HI}}$ is the neutral hydrogen fraction, $\delta$ is the matter density contrast, $x_{\text {tot }}=x_{c}+x_{\alpha}$ is the sum of the coupling coefficients which includes collisional coupling $\left(x_{c}\right)$ and the WF coupling due to Ly $\alpha$ photons $\left(x_{\alpha}\right), T_{\mathrm{CMB}}$ is the CMB temperature and $T_{\text {gas }}$ is the (kinetic) gas temperature. There are two other effects that we include in our simulations but omit from Eq. (1) in order to simplify the discussion here. One is the effect of peculiar velocities (Bharadwaj \& Ali 2004; Barkana \& Loeb 2005a); while they produce line-of-sight anisotropy that can be used for model-independent inferences of early cosmic history (Fialkov et al. 2015), in this paper we focus on the more easily measured spherically-averaged $21-\mathrm{cm}$ power spectrum. The other effect is that of low-temperature corrections to Lya scattering Chuzhov 2006; Chen \& Miralda-Escudé 2004; Hirata 2006; (Pritchard \& Furlanetto 2006; Barkana 2016).

Eq. (11) contains four different terms that can source fluctuations in the total brightness temperature:

(i) Fluctuations in the matter density affect the signal via the matter density contrast, $\delta$, defined as $\delta=\rho / \bar{\rho}-1$, where $\rho$ is the local density and $\bar{\rho}$ is the mean density; they also produce peculiar velocity fluctuations, determined by the density fluctuations through the continuity equation. Thus, we usually show the sum of the two contributions and denote it $\delta+v$; the variance of this sum is $28 / 15$ times that of $\delta$ alone (but the cross-correlation with other 21-cm fluctuations is more complicated).

(ii) The term $x_{\text {tot }} /\left(1+x_{\text {tot }}\right)$ depends on the total coupling coefficient and fluctuates due to inhomogeneous collisions at the highest redshifts and the non-uniform production of Ly $\alpha$ photons at later times.

(iii) The term $1-T_{C M B} / T_{\text {gas }}$ varies due to inhomogeneous heating.

(iv) The inhomogeneous process of reionization is encoded in the neutral fraction, $x_{\mathrm{HI}}$.

As with the definition of $\delta$, we define contrasts for each one of the other three terms, $\delta_{\text {coup }}, \delta_{\text {heat }}$ and $\delta_{\text {ion }}$, respectively. To first order, different sources of fluctuations are additive (although they may have different signs), and at each redshift the contrast in brightness temperature is approximately

$$
\delta_{T_{b}} \approx(\delta+v)+\delta_{\text {coup }}+\delta_{\text {heat }}+\delta_{\text {ion }}
$$

In reality there are also non-linear terms, and crosscorrelations contribute to the power spectrum, but it is useful to look at the separate contributions of the sources of fluctuations in order to understand which of them dominate at any given time.

It is often more convenient to discuss fluctuations in Fourier (rather than real) space, in terms of the comoving wavenumber, $k$, which is inversely proportional to the comoving scale. The total power spectrum $P_{T_{b}}(k)$ is defined by:

$$
\left\langle\tilde{\delta}_{T_{b}}(\mathbf{k}) \tilde{\delta}_{T_{b}}^{*}\left(\mathbf{k}^{\prime}\right)\right\rangle=(2 \pi)^{3} \delta_{D}\left(\mathbf{k}-\mathbf{k}^{\prime}\right) P_{T_{b}}(k),
$$

where $\tilde{\delta}_{T_{b}}(\mathbf{k})$ is the Fourier transform of $\delta_{T_{b}}, \mathbf{k}$ is the comoving wavevector, $\delta_{D}$ is the Dirac delta function, and angle brackets denote the ensemble (or spatial) average. Finally, we use the convention of expressing the power spectrum in terms of the variance in $\mathrm{mK}^{2}$ units:

$$
\Delta^{2}=\left\langle T_{b}\right\rangle^{2} \frac{k^{3} P_{T_{b}}(k)}{2 \pi^{2}},
$$

where the expression $k^{3} P_{T_{b}}(k) / 2 \pi^{2}$ is dimensionless. Because the universe is homogeneous and isotropic on large cosmological scales, the fluctuations tend to decrease with increasing scale (decreasing wavenumber).

For a typical set of astrophysical parameters, fluctuations in the $21-\mathrm{cm}$ signal on large cosmological scales, e.g., $k \sim 0.1 \mathrm{Mpc}^{-1}$, and in the redshift range $5 \lesssim$ $z \lesssim 35$ exhibit three distinct peaks (e.g., Barkana \& Loeb 2005b; Pritchard \& Furlanetto 2007; Pritchard \& Loeb 2008; Fialkov \& Barkana 2014). Prior to significant star formation, the $21-\mathrm{cm}$ brightness temperature is driven by interatomic collisions and interactions with the CMB photons. While collisions dominate at the highest redshifts, driving the 21-cm brightness temperature to the kinetic temperature of the IGM, thermal equilibrium with the CMB takes over once the universe expands enough to render collisions inefficient. When the first stars form in rare peaks of the density field and create an inhomogeneous Ly $\alpha$ background, WF coupling becomes efficient. Fluctuations are induced by the Ly- $\alpha$ peak around $z \sim 25$ (when $x_{\text {tot }} \sim x_{\alpha} \sim 1$ ) and disappear once the coupling saturates (i.e., $x_{\text {tot }} \sim x_{\alpha} \gg 1$ ). At the same time, the X-ray background builds up, leading to an increase in the temperature of the gas. This non-uniform heating creates fluctuations with a peak power at redshift $\sim 15$. When heating becomes saturated $\left(T_{\text {gas }} \gg T_{\mathrm{CMB}}\right)$ the signal no longer depends on the gas temperature. Fluctuations at low redshift $(z \sim 10)$ are dominated by patchy reionization. However, as we show below, this overall, standard picture is not universal, and the power spectrum as a function of redshift can have various numbers of peaks (between one and three) depending on the scale and on the particular choice of astrophysical parameters.

\subsection{The parameter space}

Existing observational evidence and theoretical arguments place very weak constraints on the astrophysical properties of the first luminous objects, which translates into a large uncertainty in the predicted 21-cm signal. For instance, Cohen et al. (2016b) showed that the current astrophysical parameter space yields global 21-cm spectra with the depth of the absorption trough feature anywhere in the $-250 \mathrm{mK} \lesssim T_{b} \lesssim-25 \mathrm{mK}$ range. Here we explore the implications of this large parameter space for the $21-\mathrm{cm}$ power spectrum.

We first review the relevant parameters (same as in Cohen et al. 2016b, where complete details are given) and quote the range within which they are allowed to vary:

- The star formation efficiency is the fraction of gas 
in dark matter halos that is converted into stars, $f_{*}$. In general, this fraction depends on halo mass and the cooling channel through which stars form. The efficiency measured in numerical simulations shows a large scatter, especially for the light halos that dominate the early universe (Wise et al. 2014; O'Shea et al. 2015; Xu et al. 2016). Our parameter $f_{*}$ is actually the star formation efficiency in large halos (above the atomic cooling threshold), where for smaller halos we consider two different dependencies of $f_{*}$ on the halo mass: (i) a constant down to the minimum mass and (ii) a gradual low-mass cutoff. We vary the star formation efficiency from $0.5 \%$ to $50 \%$, with $5 \%$ being our fiducial value.

- The minimum halo mass for star formation, $M_{\min }$, discussed earlier in the Introduction, can be expressed in terms of the circular velocity, $V_{c}$. Here we entertain a few possibilities: star formation in molecular cooling halos (i.e., down to $V_{c}=4.2 \mathrm{~km} \mathrm{~s}^{-1}$, affected by LW feedback and the streaming velocity), atomic cooling halos $\left(V_{c}=16.5\right.$ $\left.\mathrm{km} \mathrm{s}^{-1}\right)$, via metal line cooling $\left(V_{c}=4.2 \mathrm{~km} \mathrm{~s}^{-1}\right.$ without LW feedback but with the streaming velocity), or, finally, in massive or super-massive halos (e.g., due to strong supernovae feedback; a minimum $V_{c}=35.5$ or $76.5 \mathrm{~km} \mathrm{~s}^{-1}$, respectively). We note that since both cooling and internal feedback depend on the depth of the potential, which is measured by $V_{c}$, it is more physical to assume a fixed $V_{c}$ with redshift rather than a fixed $M_{\min }$.

- In order to accommodate the variety of X-ray sources we use two quite different X-ray SEDs that bracket a large range: (i) the commonly used soft power-law spectrum (Furlanetto 2006), and (ii) a hard spectrum that corresponds to XRBs (Fragos et al. 2013; Fialkov et al. 2014a). In addition, we consider mini-quasars (sources expected to have a hard SED similar to that of XRBs, e.g., Tanaka et al. 2012). The evolution in time of a population of mini-quasars is different from X-ray binaries, i.e., with a much later build-up of the X-ray intensity, since the X-ray luminosity has an extra dependence on the halo mass (assuming a similar relation between black hole and halo mass as observed at low redshift).

- The X-ray efficiency, $f_{X}$, accounts for the uncertain normalization of the $L_{X}$-SFR relation. We define $L_{X} / S F R=3 \times 10^{40} f_{X}\left[\operatorname{erg~s}^{-1} \mathrm{M}_{\odot}^{-1}\right.$ yr] for the cases of XRBs or the soft SED (Fialkov et al. 2014a). For the miniquasars the $L_{X}$-SFR relationship depends on both the redshift and halo mass. Our fiducial value is $f_{X}=1$, but we vary it between zero and a few hundred. A negligible amount of X-rays is not yet ruled out for some models, while the upper limit (which is model-dependent) is determined by saturating the unresolved soft X-ray background observed by Chandra in the $0.5-2 \mathrm{keV}$ band (Lehmer et al. 2012; Fialkov et al. 2017).

- Finally, we vary the CMB optical depth, $\tau$. Our fiducial value is $\tau=0.066$, which corresponds to the most recent measurement of the optical depth when we began this project (Planck Collaboration et al. 2016b). Given the rather large uncertainty in the measured value, we also consider higher values of $\tau$, including values of $\tau>0.09$ that now seem unlikely. To get the desired optical depth we vary the ionizing efficiency for each case as described in Cohen et al. (2016b).

As we will see below, the shape and redshift evolution of the power spectrum varies greatly among various sets of plausible astrophysical parameters. For instance, the difference between two otherwise identical models but with a hard or soft X-ray SED, as discussed in the next Section, is dramatic (see also Fialkov et al. 2014a; Fialkov \& Barkana 2014). In order to fully explore the effect of these parameters on the expected $21-\mathrm{cm}$ signal we run our simulation for 193 different combinations of the five parameters described above (the full list of cases appears in Appendix (A). Except for a few cases, all the considered parameters are well within the limits established by recent $21-\mathrm{cm}$ power spectrum data (Ali et al. 2015; Pober et al. 2015) and CMB data (Planck Collaboration et al. 2016b), and do not saturate the unresolved soft X-ray background (Lehmer et al. 2012).

\section{CASE STUDY}

In order to establish some intuition we begin with a relatively simple case, and examine in detail the predictions for a particular case (which we refer to as our standard), which assumes the atomic cooling minimum mass, $f_{*}=0.05$, $f_{X}=1, \mathrm{XRBs}$, and $\tau=0.066$ (\#53 in Appendix A). We demonstrate how much varying the X-ray SED affects predictions by comparing this model to case \#55 which has a soft X-ray SED but otherwise identical parameters.

The evolution of the power spectra is inherently a function of two dimensions, as the power spectrum depends on both frequency/redshift and scale. We show this full dependence in 2-dimensional color plots (top panels of Fig. 1). At different stages of cosmic history the total power in the $21-\mathrm{cm}$ fluctuations can vary anywhere between 0.001 and $1000 \mathrm{mK}^{2}$, with strong fluctuations shown in red and weak fluctuations in blue. For our standard case, WF coupling turns on at around $z \sim 30$ when the first significant population of stars appears, creating an inhomogeneous Ly $\alpha$ background which imprints a broad peak in the $21-\mathrm{cm}$ spectrum at $z \sim 20$. The effect of inhomogeneous heating is visible later at $z \sim 15$, while the signature of reionization dominates at $z \sim 10$.

Predictions of the model \#55 are the same except for the effect of X-rays, which imprints a strong signature in the intermediate redshift range and on large scales (Fialkov \& Barkana 2014). Consider first the small-scale regime $\left(k \gtrsim 0.5 \mathrm{Mpc}^{-1}\right)$. On these scales, which are below the typical mean free path of X-ray photons for both the hard and the soft SED, heating fluctuations are washed out (although more so in the hard SED case, where there is a clear drop in the power spectrum at the heating transition), and we only see two high power regions caused by (i) inhomogeneous Ly $\alpha$ radiation at the high-redshift end and (ii) ionizing fluctuations at the low-redshift end. In both cases, the signature of reionization first appears at the small-scale end and propagates to larger scales at lower redshifts, as the ionized bubbles grow. The qualitative difference between the two maps is on large scales $\left(k<0.5 \mathrm{Mpc}^{-1}\right)$ that exceed the mean free path of a typical X-ray photon in the case of the soft SED, but are still below the mean free path of photons in the hard SED case. (Note that at $z \sim 20$ the mean free path is longer than $100 \mathrm{Mpc}$ for photons with energies above $\sim 1 \mathrm{keV})$. As a result, X-ray heating imprints a strong peak 


\section{Standard}
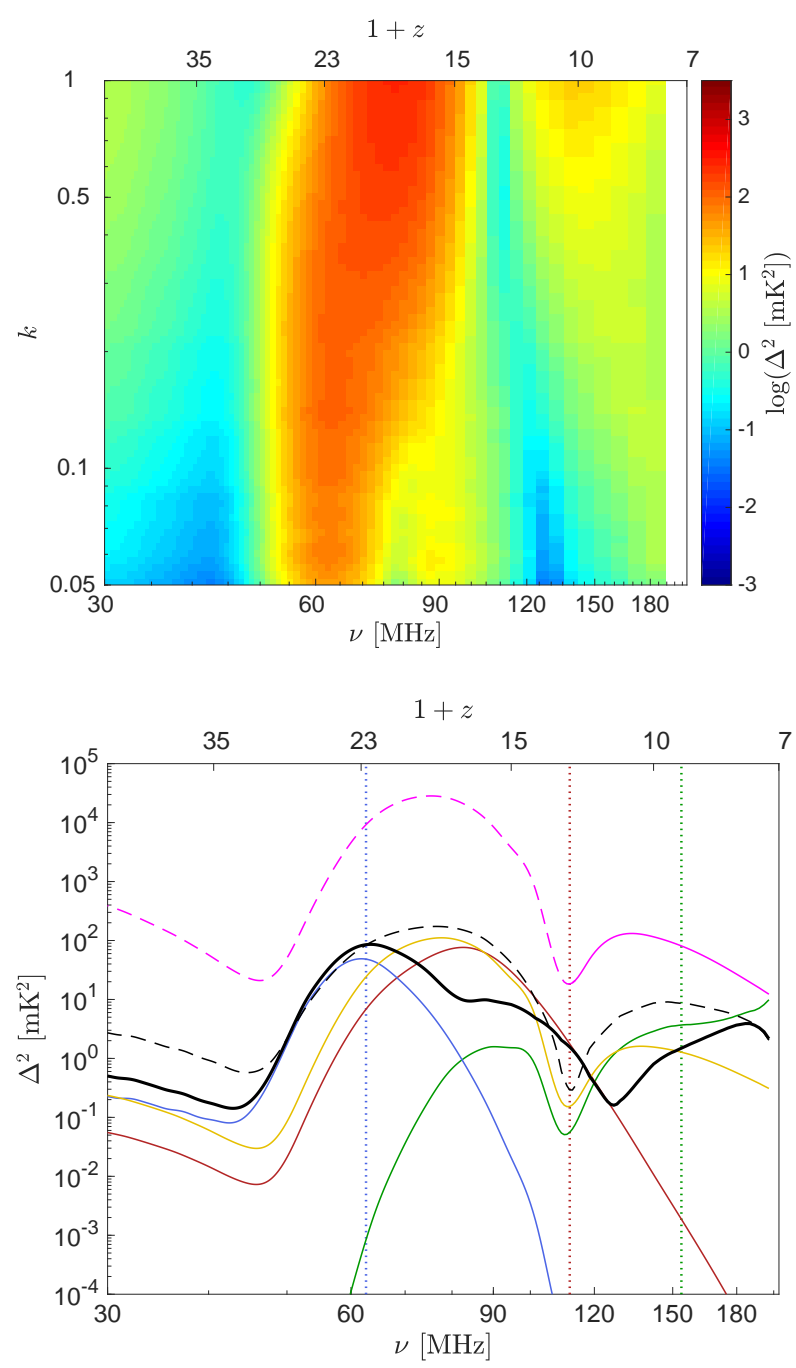

Soft SED
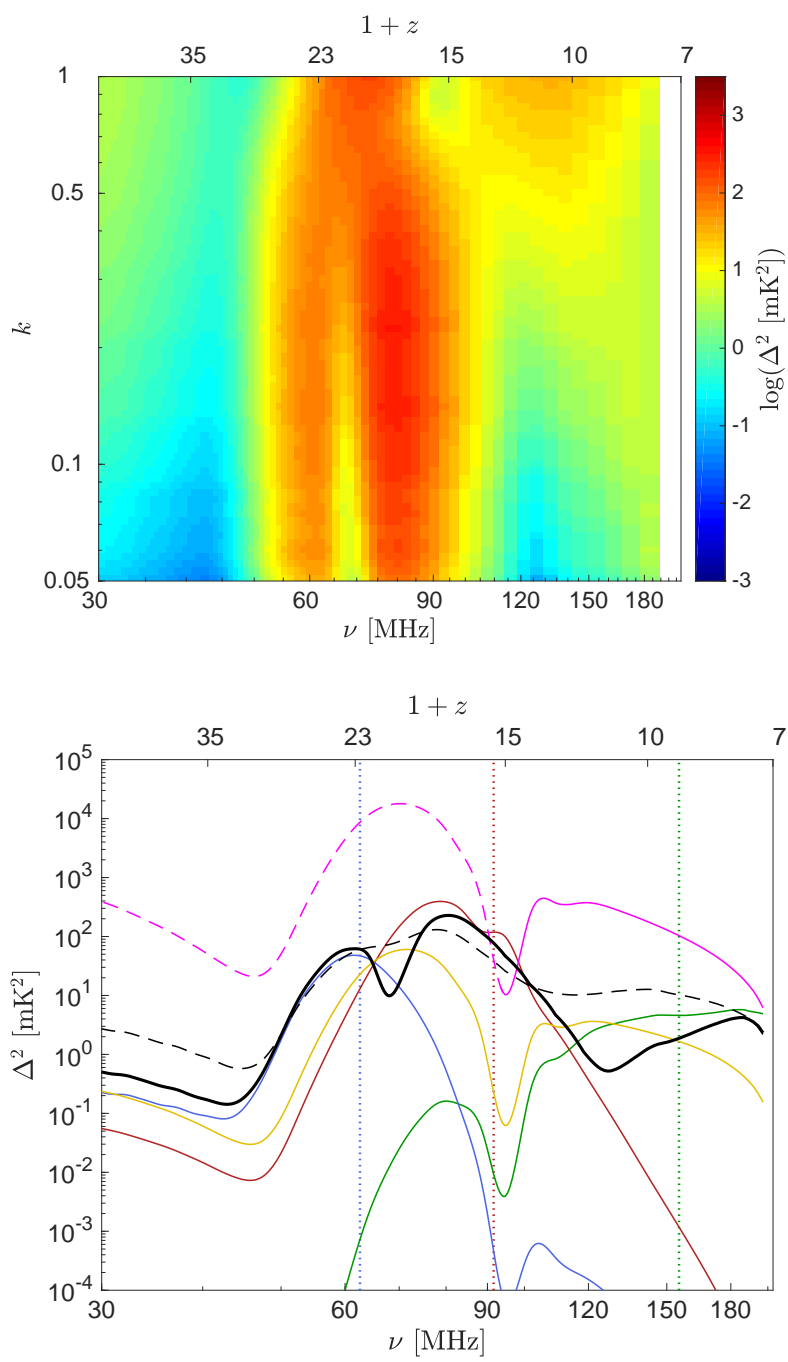

Figure 1. 21-cm power spectra for the model \#53 (our standard case, left column) and model \#55 (right column). Top panels: Twodimensional map of the power spectra as a function of both wavenumber (vertical axis) and frequency/redshift (lower/upper horizontal axis). Colors correspond to $\log \left(\Delta^{2}\right)$ as indicated on the color bar. Bottom panels: Evolution of the total power spectrum at a fixed wavenumber is shown for $k=0.1 \mathrm{Mpc}^{-1}$ (solid black) and $k=0.5 \mathrm{Mpc}^{-1}$ (dashed black) as a function of frequency/redshift (lower/upper horizontal axis). For $k=0.1 \mathrm{Mpc}^{-1}$ we also show the separate power spectra of various components: Ly $\alpha$ (blue), density plus velocity $\left(\delta+v\right.$; yellow), temperature/heating (dark red) and ionization (green); these have all been expressed like $\Delta^{2}$ [Eq. 4, in mK ${ }^{2}$ units. For comparison, the global 21-cm signal, $T_{b}^{2}$, is also shown (magenta), as dashed when the signal is negative and solid when it is positive. Vertical lines mark several important milestones, namely Ly $\alpha$ coupling (blue), the cosmic heating transition (dark red) and the midpoint of reionization (green).

in the former case, compared to a barely-noticeable peak (and only at $k=0.1 \mathrm{Mpc}^{-1}$ and below) in the latter case.

A brief note on scales: a half-wavelength equal to our pixel size (corresponding to the Nyquist critical frequency) is $k \sim 1 \mathrm{Mpc}^{-1}$. At the other end, a half-wavelength equal to the size of our box corresponds to $k \sim 0.008 \mathrm{Mpc}^{-1}$, but even $k$ values that are a few times that suffer from being averaged over only a small number of samples (as we have verified by comparison to larger simulation boxes). Thus, in order to avoid edge effects at both ends, we only use the range $k=0.05-1 \mathrm{Mpc}^{-1}$, and focus on the specific values $k=0.1 \mathrm{Mpc}^{-1}$ (which we refer to as representing large scales) and $k=0.5 \mathrm{Mpc}^{-1}$ (small scales).
To follow the evolution of fluctuations with redshift more closely, it is useful to examine the behavior of the total power spectrum at a fixed comoving scale, which we show in the bottom panels of Fig. 1 for $k=0.1 \mathrm{Mpc}^{-1}$ (solid black, representing large scales) and $k=0.5 \mathrm{Mpc}^{-1}$ (dashed black, representing small scales). In agreement with the color plot, on large scales the case with a soft SED shows three separate peaks dominated by Ly $\alpha, \mathrm{X}$-ray and ionization fluctuations, respectively; while the other (hard SED) case has only two peaks, dominated by $\operatorname{Ly} \alpha$ and by ionization fluctuations. In the latter case X-rays contribute only a knee around $z \sim 15$. In the small-scale regime the signatures of a soft and hard SED are more similar: the gas temperature 
is nearly uniform on such scales in both cases, and there is no heating peak. In both of these cases there are two peaks in the absolute value of the global 21-cm temperature, one in absorption (when the Ly $\alpha$ coupling saturates and X-ray heating first becomes significant), and one in emission (after heating saturates); they are separated by a minimum near the cosmic heating transition (though $k=0.5 \mathrm{Mpc}^{-1}$ is a borderline value in the soft SED case, a scale large enough that the temperature fluctuations are significant and nearly eliminate this minimum). This naturally tends to produce two redshift peaks in the 21-cm power spectrum as well (at a given $k$ ), although extra astrophysics (such as a rise and fall of various sources of fluctuations) can move the peaks or create additional ones.

It is interesting to examine in detail what is the leading source of fluctuations at every epoch. To explore this aspect we directly extract from our simulation and separately show on the same plot (bottom panels of Fig. 1) power spectra (normalized as in Eq. (4)) of each of the terms $\delta+v, \delta_{\text {coup }}, \delta_{\text {heat }}$ and $\delta_{\text {ion. }}$. The total power spectrum is then a sum of individual contributions of power spectra from each type of fluctuations plus cross-correlations among the various sources. Note that unlike the power spectra of each component (which are positive by definition), the cross-correlation components of the power spectrum can be negative. In particular (Fialkov \& Barkana 2014), density fluctuations correlate positively with Ly $\alpha$ fluctuations and anti-correlate with ionization fluctuations; density fluctuations anti-correlate with heating fluctuations when the gas is colder than the CMB, and correlate positively with heating fluctuations when the gas is hotter than the CMB. In the plots we only show the power spectra of each of the sources of fluctuations separately. This allows us to see which fluctuation source dominates at each redshift. For the specific choice of astrophysical parameters shown, the coupling term (blue curve) dominates at high redshifts, around the Ly $\alpha$ coupling transition (defined as the redshift at which $x_{\text {tot }}=1$; vertical blue dotted line. Note that our plots also include the dark ages $(z>30)$ but we do not focus on them here). This contribution starts to fade when Ly $\alpha$ coupling saturates $\left(x_{\text {tot }} \gg 1\right)$. Next, the build-up of the X-ray radiation background leads to a rise in fluctuations in the $1-T_{C M B} / T_{g a s}$ term (dark red curve). All contributions except for heating experience a minimum around the cosmic heating transition (defined as the redshift at which the mean IGM temperature equals the CMB temperature; vertical dark red dotted line. Note that this can be offset by a $\Delta z \sim 0.5$ compared to the redshift at which the mean $T_{b}=0$.). Heating fluctuations disappear once the IGM temperature is well above the CMB temperature. Finally, the ionization fluctuations dominate (solid green line) around the midpoint of reionization (defined as when the cosmic mean mass-weighted $x_{\mathrm{HI}}=0.5$; vertical green line).

However, this is not the full story. The contribution of density (plus velocity) fluctuations, which is often considered to be sub-dominant relative to the radiative contributions, is significant throughout cosmic history from the dark ages to the end of reionization. As is evident from the plots, at some moments in cosmic history, particularly when a dominant radiative contribution fades away, density fluctuations can dominate the total power of the 21-cm signal. This usually occurs in-between peaks, but as we go towards smaller scales (including $k=0.5 \mathrm{Mpc}^{-1}$ ), it becomes more common for density fluctuations to be the dominant factor at a peak of the power spectrum.

It is easy to understand the redshift evolution of the density term. Because the density power spectrum evolves slowly with redshift in the linear regime, $P_{\delta} \propto(1+z)^{-2}$, the redshift evolution of the density (plus velocity) contribution $\left(\Delta_{\delta}^{2} \propto T_{b}^{2} \times P_{\delta}\right.$, yellow curve) is largely driven by $T_{b}^{2}$ (shown with a magenta line on the plot, for comparison). Note that the difference between $T_{b}^{2}$ and the density contribution decreases at low redshifts because of the growth of the density contrast.

Since the power spectrum is a function of two variables ( $k$ and $z$ ), we next consider the shape of the power spectrum (see Fig. 2). Specifically, the spectral index of the power spectrum is commonly defined as $\beta \equiv \frac{d \ln \Delta^{2}}{d \ln k}$. Naturally, if the spectral index is positive, fluctuations have more power on smaller scales (larger $k$ ) and if it is negative, there is more power on larger scales (smaller $k$ ). If the spectrum is a pure power law, it has a shape $\Delta^{2} \propto k^{\beta}$ with constant $\beta$. In the $21-\mathrm{cm}$ case, there are several sources of fluctuations that drive the signal $\left(\delta+v, \delta_{\text {coup }}, \delta_{\text {heat }}\right.$ and $\left.\delta_{\text {ion }}\right)$, each having its own spectral index that can be either positive or negative. In particular, in the hierarchical picture of structure formation density fluctuations have more power on small scales and, therefore, $\beta_{\delta}>0$. The coupling term has a more complex temporal evolution. During the dark ages $\delta_{\text {coup }}$ is dominated by the collisional term which follows $\delta$ on all scales and thus we expect a positive spectral index $\beta_{\text {coup }}=\beta_{\delta}$. However, as soon as Ly $\alpha$ coupling becomes dominant, $\beta_{\text {coup }}$ should drop (and may even become negative) on scales below the mean free path (which is of order tens of comoving Mpc for Ly $\alpha$ photons). This is since fluctuations are washed out on such scales. However, on scales much larger than the mean free path, fluctuations continue to mostly follow the density and maintain a positive spectral index. Realistically, the transition between large and small (or negative) spectral indexes happens smoothly versus redshift. Similarly, heating fluctuations are first driven by the density and have $\beta_{\text {heat }}=\beta_{\delta}$, while at later times (once X-ray heating dominates) the power spectrum of $\delta_{\text {heat }}$ flattens. Finally, in the inside-out picture of reionization (Barkana \& Loeb 2004), fluctuations in the ionizing field are roughly a convolution of the density field with a bubble of a typical size at each redshift. We expect the spectral index to be close to $\beta_{\delta}$ on scales larger than the average size of an ionized bubble (which grows with time), and to drop on smaller scales. The total power spectrum is a combination of the four terms and is driven by one or sometimes a few terms at a time. Therefore, its spectral index evolves with redshift and is scale-dependent, as can be seen in Fig. 2 where the power spectrum of our standard case (which has a hard SED) is shown as a function of $k$ at several redshifts.

Rather than using the scale-dependent spectral index, we define a simpler measure that captures the essence of the $k$ dependence, namely, the average slope of the power spectrum between two scales (still in log-log):

$$
B=\frac{\ln \Delta^{2}\left(k_{2}\right)-\ln \Delta^{2}\left(k_{1}\right)}{\ln k_{2}-\ln k_{1}} .
$$

In Fig. 3 we show the evolution of the slope with redshift, both in the small-scale regime (calculated between $k_{1}=0.2$ 

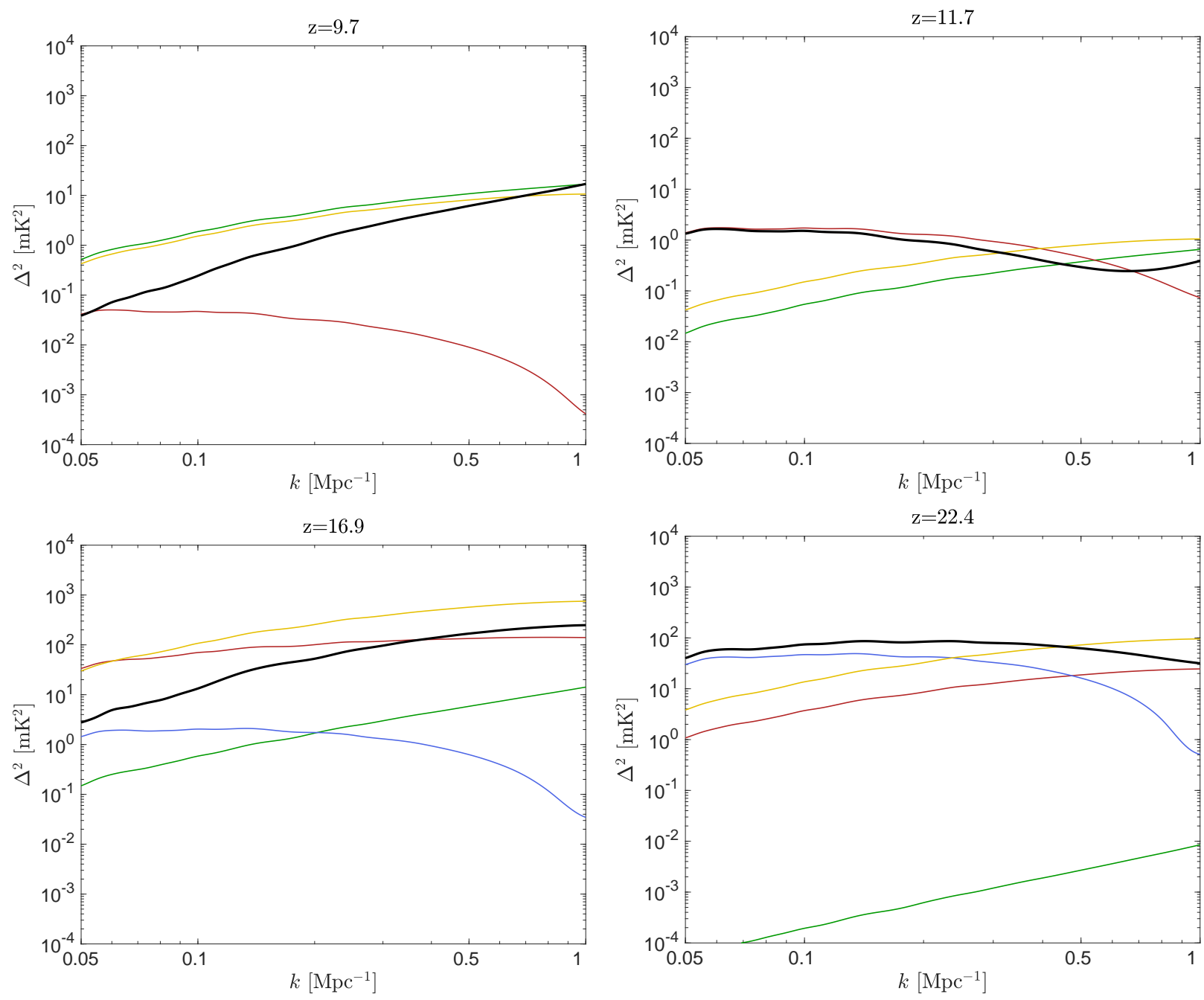

Figure 2. The 21-cm power spectrum (black) in our standard case as a function of $k$ at redshifts $z=9.7,11.7,16.9$ and 22.4 (see panel titles) corresponding to the extrema of the large-scale slope as seen in Fig. 3 Also shown are the power spectra of the components $\delta+v$ (yellow), $\delta_{\alpha}$ (blue), $\delta_{\text {heat }}$ (dark red) and $\delta_{\text {ion }}$ (green). At $z=9.7$ and 16.9 the total power spectrum shows a near-cancellation of different components with opposite signs.

$\mathrm{Mpc}^{-1}$ and $k_{2}=0.6 \mathrm{Mpc}^{-1}$, corresponding to $\sim 10-30$ comoving $\mathrm{Mpc}$ ) and in the large-scale regime (calculated between $k_{1}=0.05 \mathrm{Mpc}^{-1}$ and $k_{2}=0.2 \mathrm{Mpc}^{-1}$, corresponding to $\sim 30-125$ comoving Mpc). In addition to the slope of the total power spectrum, $B_{\text {tot }}$, we show the slopes for each of the four sources of fluctuations, $B_{\delta}, B_{\text {coup }}, B_{\text {heat }}$, and $B_{\text {ion }}$.

The overall evolution of the spectral slope, as outlined above, can be seen in detail in Fig. 3. First of all, $B_{\delta}$ is positive and constant with redshift (because in the linear regime within $\Lambda$ CDM cosmology, the growth of density fluctuations is scale-independent); $B_{\delta} \sim 3$ where the matter power spectrum turns over (i.e., at $k_{\text {eq }} \sim 0.06 \mathrm{Mpc}^{-1}$ ), and decreases with $k$. Both $B_{\text {coup }}$ and $B_{\text {heat }}$ start out equal to $B_{\delta}$, while at later times (when they become dominated by astrophysical radiation) they drop to zero or below (on scales below the effective horizon of the radiation). The evolution of $B_{\text {ion }}$ is interesting, as it has a particularly high peak value at very high redshifts. This is because the non-linear amplification (i.e., the fact that the ionization fraction is either 0 or 1 ) increases the power on small scales, in particular causing large Poisson fluctuations initially (Barkana 2008), for which we would expect a slope around 3; this, though, mostly occurs long before there is enough reionization to significant affect the total $21-\mathrm{cm}$ power spectrum. At lower redshifts, the ionized bubbles grow and wash out small-scale fluctuations in the ionization.

The slope of the total $21-\mathrm{cm}$ power spectrum is driven by one or several components at a time and its evolution can be easily understood (as we illustrate with the large-scale slope of our standard case). During the cosmic dark ages, all fluctuations are driven by the density, and so $B_{\text {tot }}=B_{\delta}$. When the first significant population of stars creates a Ly $\alpha$ background, the power spectrum flattens and its slope decreases as $B_{\text {tot }}$ follows $B_{\text {coup }}$. In fact, $B_{\text {tot }}$ of the large-scale slope has a minimum just when the contribution of Ly $\alpha$ to the power spectrum, shown in Fig. 1] is maximal $(z=22.4$ in the standard case). Then, when $x_{\alpha}$ approaches saturation, the contribution of Ly $\alpha$ fluctuations becomes negligible compared to density fluctuations. As a result, the total power spectrum steepens again and the slope increases. $B_{\text {tot }}$ peaks when $\delta_{\text {heat }}$ is comparable to $\delta+v$, causing cancellation on large scales, but not on small scales (where the heating 

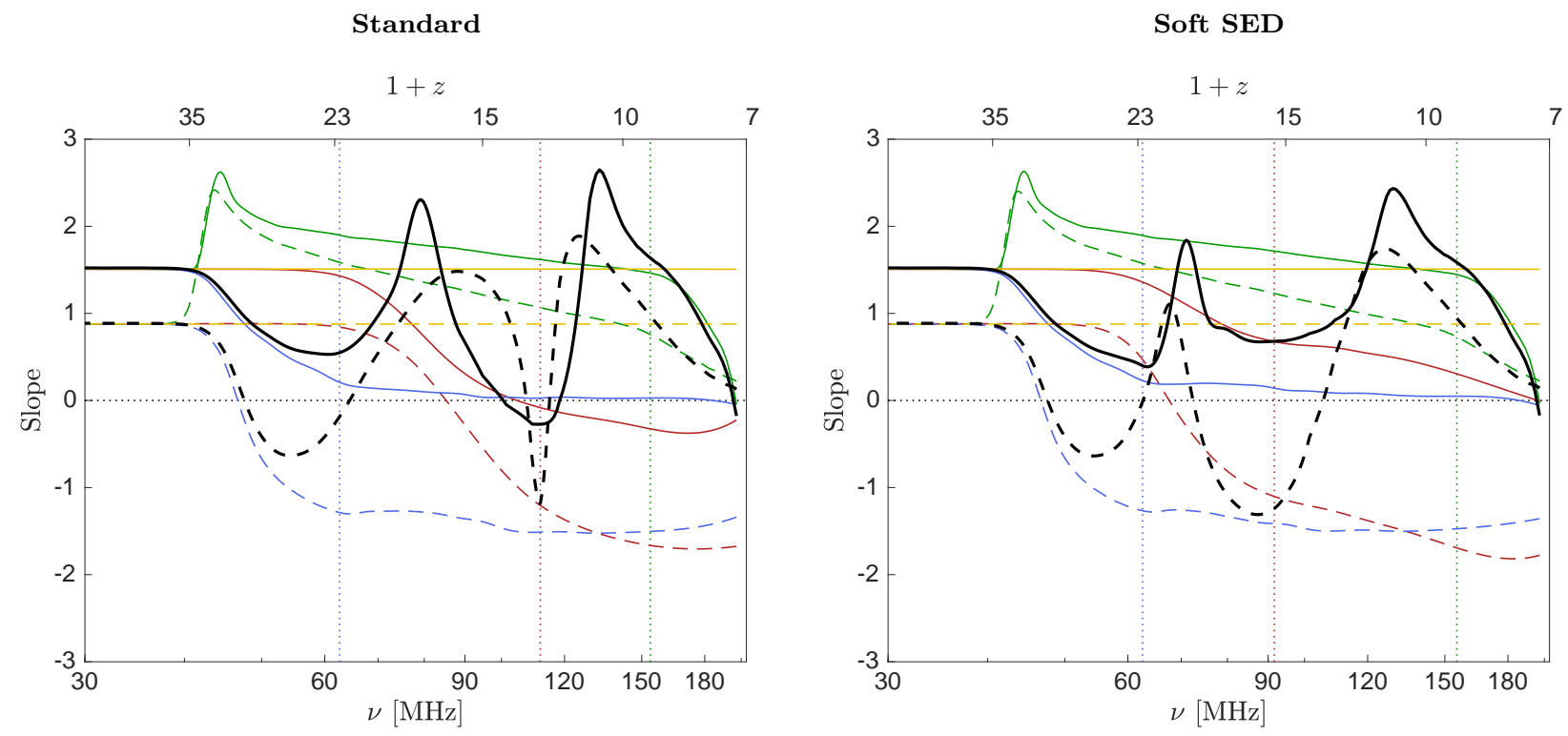

Figure 3. Slopes are shown for the standard case (left) and the case with soft SED (right) at large scales (between $k=0.05$ and 0.2 $\mathrm{Mpc}^{-1}$, solid) and small scales (between $k=0.2$ and $0.6 \mathrm{Mpc}^{-1}$, dashed) as a function of frequency/redshift (lower/upper horizontal axis). We show $B_{\text {tot }}$ (black), $B_{\delta}$ (yellow), $B_{\text {coup }}$ (blue), $B_{\text {heat }}$ (red) and $B_{\text {ion }}$ (green).

power spectrum is flatter); see Fig. 2 at $z=16.9$. At the heating transition $(z=11.7)$, heating fluctuations dominate on large scales (Fig. 2); therefore, $B_{\text {tot }}$ reaches a minimum as it tracks $B_{\text {heat }}$. After the heating transition is completed and the IGM becomes much hotter than the CMB, the impact of heating on the $21-\mathrm{cm}$ signal gradually declines. As a result, for a short time the density fluctuations become the main source of the $21-\mathrm{cm}$ power again. At the same time, ionizing fluctuations become increasingly important and eventually win, surpassing the contribution of density at $z=10.8$. The slope peaks at $z=9.7$ when ionization fluctuations become large enough to cancel out the sum of density (plus velocity) and heating fluctuations, with heating being significant only on very large scales and thus the small-scale fluctuations do not cancel out (Fig. 2). After that, ionization fluctuations dominate the 21-cm fluctuations. Around the midpoint of reionization (vertical green line), there is an inflection point in $B_{\text {tot }}$, as the main source of fluctuations changes from ionized bubbles within a neutral IGM to remaining neutral regions within a mostly ionized IGM. $B_{\text {tot }}$ tracks $B_{\text {ion }}$ during the later stages of reionization.

The general behavior of the small-scale slope (dashed black line) is similar to that of the large scale slope. However, the numerical value of this slope is generally lower because the density power spectrum has a lower slope on small scales. Also, both Ly $\alpha$ and X-rays are types of long-range radiation and have lower slopes on small scales than the density. As a result, the two minima of the slope are significantly lower (-0.63 and -1.19 for small scales compared to 0.52 and -0.27 for the large scales).

To illustrate the effect of different astrophysical parameters on the shape of the power spectrum and its slope we show more examples in Appendix B (Figs. B1 B2 and B3) in which we vary $f_{*}, f_{X}$, and $V_{c}$ from their values in our standard case. Given the existing uncertainty in the parameters, the evolution of the power spectrum and its slope vary quite a lot among plausible parameter sets, and the change induced by varying one parameter is not a small variation around the standard case. Depending on the parameter set, there are differences in both the location and amplitude of features in the power spectrum and its slope. Moreover, as we see from the plots, the dominant sources of fluctuations at various moments in cosmic history can change, altering the history of the power spectrum and changing the number of peaks.

\section{THE ENTIRE PARAMETER SPACE}

Having considered a few specific cases in the previous section, we now address the full set of models introduced in Section 2.2. In Cohen et al. (2016b) we used these models to analyze the features of the global 21-cm spectrum (left panel of Fig. 4). The shape of this signal is universal and has three main features: (i) a high-redshift maximum at redshift $z_{\mathrm{g}, \text { max }}$ marks the onset of significant stellar radiation, (ii) a minimum (i.e., absorption trough) located at $z_{\mathrm{g}, \min }$ is the beginning of the X-ray heating era, and (iii) a high-redshift maximum (emission peak) at $z_{\mathrm{g}, \text { max }}^{\text {lo }}$ occurs when heating saturates. Here we use the same set of models to first analyze the shapes versus redshift of the power spectrum and its slope, and then relate the features to the astrophysical parameters that we vary. We summarize in Table 1 our notation for the various features of the global 21-cm signal, the power spectrum, and its slope.

\subsection{Shapes}

To demonstrate the span of possibilities that could be realized in Nature, we begin by placing all the power spectra on the same plot (right panel of Fig. 4). On every curve we mark the cosmic milestones: the redshift at which Ly $\alpha$ coupling saturates (red dot), the moment of the heating transition (green dot), and the midpoint of reionization (blue 

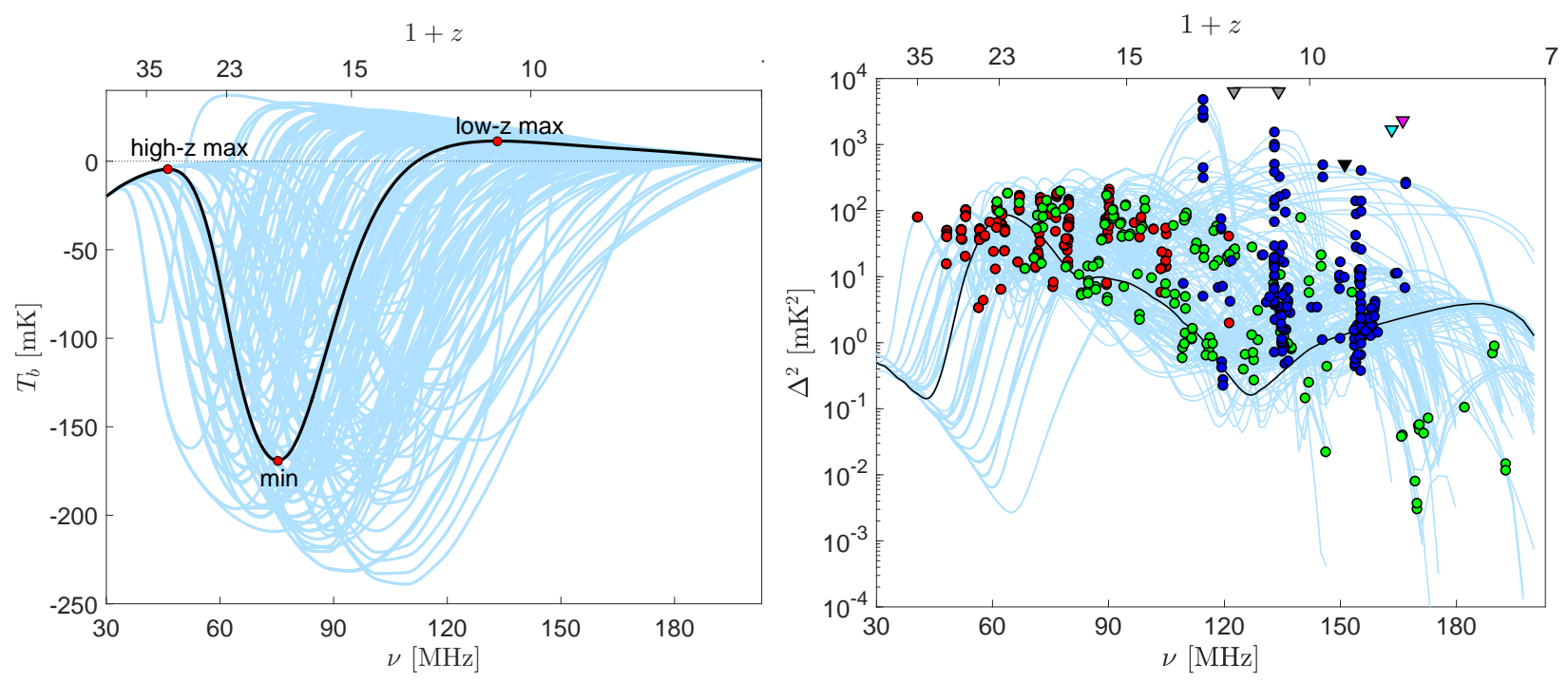

Figure 4. Left: Parameter study of the global signal adopted from Cohen et al. (2016b). We show the 21-cm global signal as a function of redshift for our standard case (black line), with red points marking the three turning points (from left to right: the high- $z$ maximum, the minimum, and the low- $z$ maximum). Light-blue lines show the entire set of realizations of the 21 -cm signal for the 193 different astrophysical models discussed in this paper. Right: The corresponding complete set of realizations of the power spectrum at $k=0.1$ $\mathrm{Mpc}^{-1}$ (light-blue curves) as a function of observed frequency/redshift (bottom/top axis). The standard case is shown with a black line. For each model, we mark the redshift of the Ly $\alpha$ coupling (red dot), the moment of the heating transition (green dot), and the midpoint of reionization (blue dot). Triangles mark various upper limits measured on various scales: black at $k=0.1$ to $0.33 \mathrm{Mpc}^{-1}\left(\mathrm{Ali}^{\mathrm{et}}\right.$ al. 2015), cyan at $k=0.18 \mathrm{Mpc}^{-1}$ (Parsons et al. 2014), magenta at $k=0.13 \mathrm{Mpc}^{-1}$ (Jacobs et al. 2015) and grey at $k=0.04 \mathrm{Mpc}^{-1}$ (Patil et al. 2017)

dot). The large scatter in the location of these markers and the large variety of shapes express our ignorance about the high-redshift astrophysical parameters. The markers of the midpoint of reionization fill a relatively small range because this transition is the most constrained, being pinned down by the Planck measurements. On the other hand, the timing of the $\operatorname{Ly} \alpha$ transition as well as the heating transition are very unconstrained and show large scatter. The former event depends on the cooling channel and the efficiency with which the first stars where formed, while the latter depends on both the properties of the first stars and of the first heating sources (which may be two significantly different populations). Note that in some cases the gas temperature does not reach the CMB temperature even at the end of reionization, due to very inefficient heating or no heating at all.

Our approach to classifying different cases is by using the properties of the peaks (which are easiest to observe), with each maximum being tagged according to the dominant source of fluctuations. The unconstrained astrophysical parameters introduce at least an order of magnitude uncertainty in the maximal power produced by each type of source. Fig. 5 shows the maxima for all the cases at $k=0.1$ $\mathrm{Mpc}^{-1}$ (left) and $k=0.5 \mathrm{Mpc}^{-1}$ (right), demonstrating the scatter in peaks dominated by density (yellow), Ly $\alpha$ (blue), heating (red) and ionization (green). Within the uncertainty introduced by the astrophysical parameters, the peak power is typically higher by $1-2$ orders of magnitude at high redshifts compared to the low-redshift ionization peak. This is an important conclusion demonstrating that the high-redshift signal might be as accessible as its lowredshift counterpart with an SKA-like instrument. On large scales $\left(k=0.1 \mathrm{Mpc}^{-1}\right)$ and prior to reionization the peak power for all the models varies in the range $5 \lesssim \Delta^{2} \lesssim 500$
$\mathrm{mK}^{2}$ and drops as soon as reionization progresses (except for the cases when reionization is cold). On smaller scales the typical peak power prior to reionization is slightly higher and is in the range $10 \lesssim \Delta^{2} \lesssim 1000 \mathrm{mK}^{2}$. It is interesting to note that, whereas on large scales all sources of fluctuations can contribute a strong peak, on scales smaller than the typical mean free path of the radiation (including $k=0.5 \mathrm{Mpc}^{-1}$ ) density fluctuations play a relatively important role, dominating the high-redshift peak (usually attributed to heating) in a significant fraction of cases. Finally we note that the number of peaks on small-scales $(1-3$, usually 2$)$ is typically smaller than on large scales $(2-3$, usually 3$)$.

As expected, the large uncertainty in the power spectrum leads to a large uncertainty in its slope (top panels of Fig. 6). It turns out that, unlike in the case of the power spectra where the number of peaks varies for different sets of parameters, in the vast majority of cases the slopes exhibit a universal shape having two maxima and two minima (as was shown for the standard case). This rule breaks down only in cases of very inefficient heating where there is no heating transition before the end of reionization, resulting in only one maximum and one minimum (e.g., left panel of Fig. B3). Another exception are the cases with super-massive halos, which show a small extra peak (bump) in the large scale slope before the high-redshift minimum (e.g., right panel of Fig. B3). The explanation of this bump is as follows. When the radiative sources first turn on (and significantly produce mainly Ly $\alpha$ ), there is a first stage where they only reach relatively short distances (due to time retardation: at the retarded time corresponding to a large distance, there were far fewer sources in existence). Thus, at this stage the radiation amplifies small-scale fluctuations, and the power-spectrum slope rises. After a short time, the radiation reaches large 

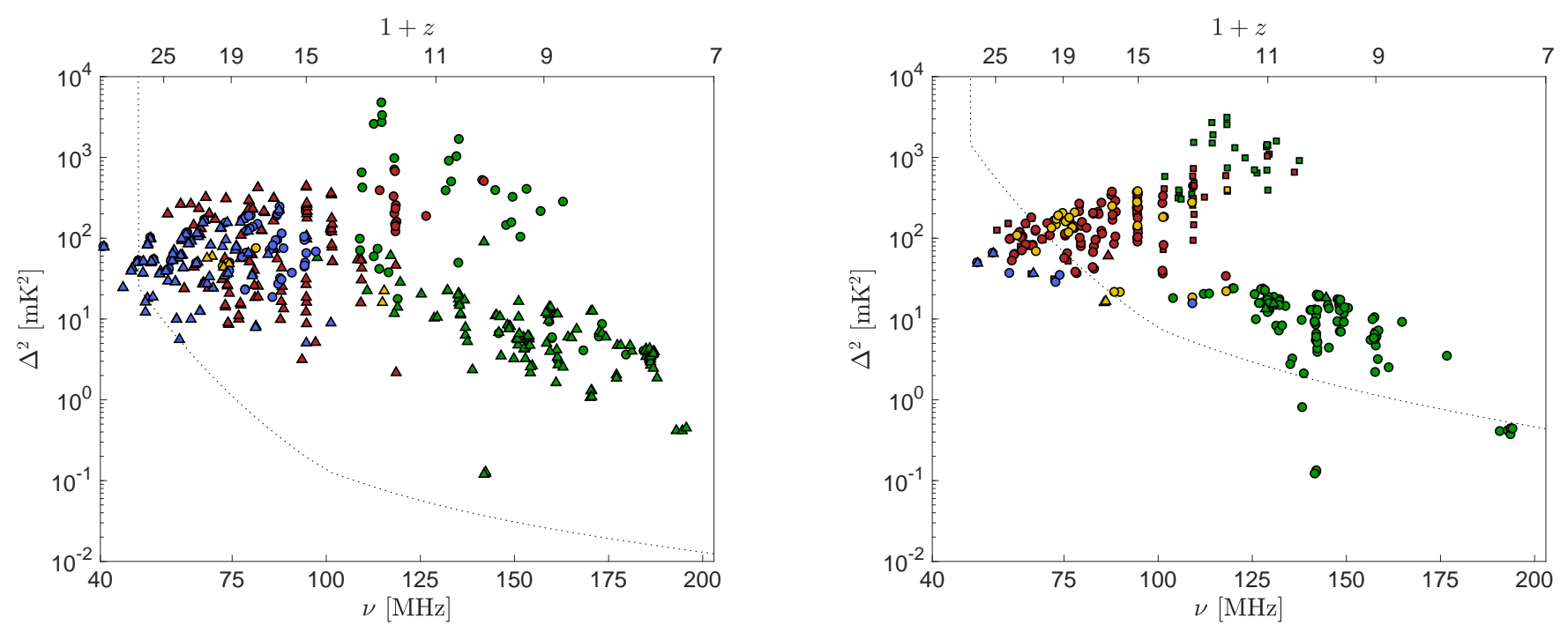

Figure 5. The power spectrum at $k=0.1 \mathrm{Mpc}^{-1}$ (left) and $k=0.5 \mathrm{Mpc}^{-1}$ (right) as a function of observed frequency/redshift (bottom/top axis) at various peaks. Marker colors indicate the dominant source of fluctuations for each peak: density (yellow), Ly $\alpha$ (blue), heating (red) and ionization (green). Marker shapes indicate whether the corresponding case has a total (over all redshifts) of 1 (squares), 2 (circles), or 3 (triangles) peaks at that scale (Note that there are no squares in the left panel). The black dotted line shows the power spectrum of the thermal noise for the SKA1 assuming a single beam, integration time of $1000 \mathrm{hours,} 10 \mathrm{MHz}$ bandwidth, and bins $\Delta k=k$.

scales and begins to smooth over the small scales, and the power-spectrum slope falls. What determines if this bump is seen in the total power-spectrum slope is whether, during this short initial period, the radiation already has a significant effect on the 21-cm signal. In most models, the Ly $\alpha$ flux turns on (and goes through this initial period) very early (at $z>30$ ), when collisional coupling is still significant, so that the Ly $\alpha$ coupling at this time is negligible. However, in models with very massive halos, the Ly $\alpha$ turn-on occurs so late (at $z<25$ ) that collisional coupling is negligible, and even the earliest Ly $\alpha$ coupling immediately dominates the 21-cm emission. Note, thought, that since the bump is only seen when the coupling is very weak, the overall height of the power spectrum at this point is very low $\left(<10^{-2} \mathrm{mK}^{2}\right)$ and thus very hard to observe.

In total, (i) 150 out of 193 cases have four extrema in the slope, including both high- and low-redshift maxima and minima, and (ii) the rest of the cases have two extrema: a high-redshift minimum and low-redshift maximum. In both type (i) and (ii) cases, a bump can additionally appear depending on $M_{\min }$. Although the exact position of each feature might vary, the overall shape with redshift of the slope is uniform (apart from the low heating cases) and allows us to correlate each of its features with specific cosmic events, as we discuss in the next subsection. The bottom panels of Fig. 6 shows the distribution of the extrema of the slopes. It is interesting to note that on the scatter plots there is little overlap in the redshift-slope plane between the areas populated by the various extrema, unlike in the case of the power spectra where there is more overlap (Fig. 5). This property should make it easier to correctly classify each feature in a slope versus redshift plot made from future observed data.

To summarize (see also Table1), the classification of the power spectra and the slopes is more difficult than that of the global $21-\mathrm{cm}$ spectra because of the diversity of features and shapes. In the case of the power spectrum we use peaks to classify each case and label the peaks according to the leading source of fluctuations $\left(z_{\mathrm{PS} \text {,density }}, z_{\mathrm{PS} \text {,coup }}, z_{\mathrm{PS} \text {, heat }}\right.$, $\left.z_{\mathrm{PS} \text {,ion }}\right)$; while for the slope we refer to the high and low minima and maxima $\left(z_{\mathrm{dPS}, \min }^{\mathrm{hi}}, z_{\mathrm{dPS}, \max }^{\mathrm{hi}}, z_{\mathrm{dPS}, \min }^{\text {lo }}, z_{\mathrm{dPS}, \max }^{\text {lo }}\right)$. Although we do show cases with $\tau>0.09$, we exclude them from all the quantitative results in the paper as those are more than $3 \sigma$ away from the optical depth measured by the Planck satellite.

\subsection{Extracting Astrophysical Properties}

As we discussed in Section 3, features of both the power spectrum and its slope are closely related to the physical properties of the early universe, as are features of the global 21-cm spectrum. Cohen et al. (2016b) related each of the observable features of the global signal to the mean astrophysical properties. In particular, the location of the highredshift maximum is closely related to $V_{c}$ and $f_{*}$ and can be translated to the mean intensity of the Ly $\alpha$ background at that redshift (Fig. 3 of Cohen et al. 2016b); the depth of the absorption trough is set by $V_{c}, f_{*}, f_{X}$ and the X-ray $\mathrm{SED}$, and correlates with the ratio of the Ly $\alpha$ intensity to the X-ray heating rate at the redshift of the minimum (Fig. 6 of Cohen et al. 2016b); while the emission peak is driven by $V_{c}, f_{*}, f_{X}$, the X-ray SED and $\tau$, and can constrain the mean heating rate over the mean ionizing efficiency (Fig. 8 of Cohen et al. 2016b). Here we use the same set of models to establish universal relationships between the features of the power spectrum (and its slope) and the astrophysical parameters.

We start by analyzing the high-redshift domain where the 21-cm signal is largely driven by the Ly $\alpha$ background. We find that for most of the considered cases, on large scales, the Ly $\alpha$ background dominates the highest redshift peak of the power spectrum. In general, increasing the typical mass of halos that dominate star formation lowers the mean Ly $\alpha$ 

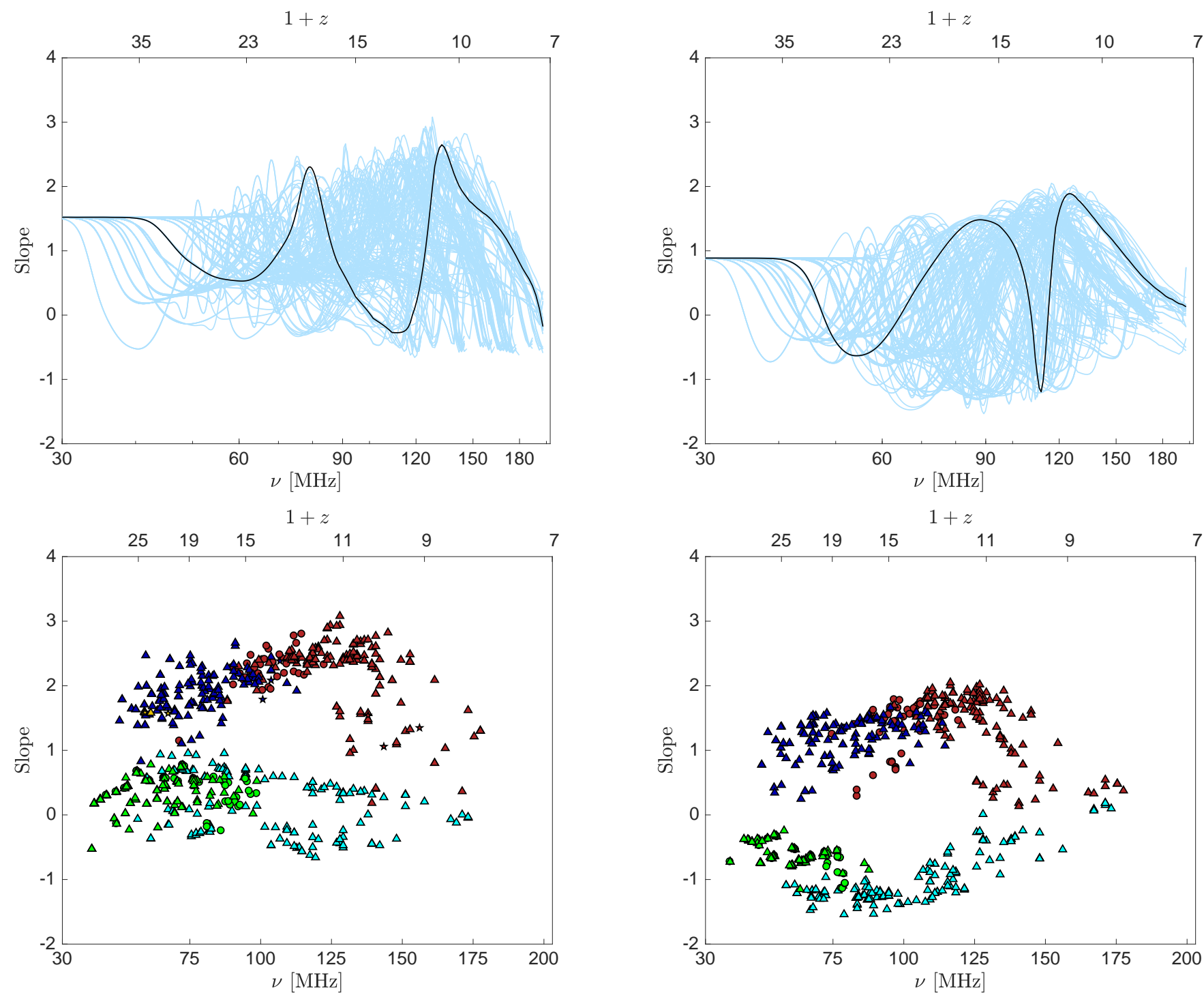

Figure 6. Top The slope of the 21-cm power spectrum on large $\left(k=0.05-0.2 \mathrm{Mpc}^{-1}\right.$, left $)$ and small $(k=0.2-0.6 \mathrm{Mpc}-1$, right $)$ scales as a function of observed frequency/redshift (bottom/top axis) for all the cases discussed in this paper (light-blue). The standard case is shown as a black line. The full list of models appears in Appendix A Bottom The extrema of the slope for large $(k=0.05-0.2$ $\mathrm{Mpc}^{-1}$; left panel) and small $\left(k=0.2-0.6 \mathrm{Mpc}^{-1}\right.$; right panel $)$ scales, presented for all cases. Marker shapes indicate the total number of minima or maxima for each case/model: 1 (circle), 2 (triangle) or 3 (star). Marker colors indicate the type of the point: red (low-redshift maximum), cyan (low-redshift minimum), blue (high-redshift maximum), green (high-redshift minimum) and yellow (extra bump; see the right panel of Fig. B3). Note that there are no stars or yellow points in the panel on the right.

\begin{tabular}{|c|c|c|c|}
\hline & Structure & Notations & Figure \\
\hline Global signal & 2 maxima and 1 minimum point & $z_{\mathrm{g}, \max }^{\mathrm{hi}}, z_{\mathrm{g}, \min }, z_{\mathrm{g}, \max }^{\mathrm{lo}}$ & 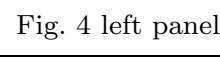 \\
\hline Power spectrum & Between 2 and 4 peaks & $\begin{array}{l}z_{\mathrm{PS}, \text { coup }}, z_{\mathrm{PS}, \text { density }}, z_{\mathrm{PS}, \text { heat }}, z_{\mathrm{PS}, \text { ion }} \\
\text { each peak classified by its dominant component }\end{array}$ & Figs. 1 and 5 \\
\hline Power spectrum slope & $\begin{array}{l}2 \text { minima and } 2 \text { maxima or } \\
1 \text { maximum and } 1 \text { minimum }\end{array}$ & $\begin{array}{l}z_{\mathrm{dPS}, \min }^{\mathrm{hi}}, z_{\mathrm{dPS}, \max }^{\mathrm{hi}}, z_{\mathrm{dPS}, \min }^{\mathrm{lo}}, z_{\mathrm{dPS}, \max }^{\mathrm{lo}} \text { or } \\
z_{\mathrm{dPS}, \min }^{\text {lo }}, z_{\mathrm{dPS}, \max }^{\text {lo }}\end{array}$ & Figs. 3 and 6 \\
\hline
\end{tabular}

Table 1. Summary of features and notations that we use to categorize the global signal, the power spectrum, and its slope. Note that the slope also sometimes has an extra small bump at very high redshift (right panel of Fig. B3). 
intensity at the redshift of the peak, and raises the powerspectrum (since massive halos are more strongly biased). However, there is a lot of overlap in the ranges (see Fig. 7), so that a given observed $\Delta_{\mathrm{PS} \text {, coup }}^{2}$ cannot be used to deduce the value of $J_{\alpha}$ without knowing the minimum mass of starforming halos. The minimum cooling mass can be estimated separately, e.g., from measuring the high-redshift maximum of the global signal (Cohen et al. 2016b).

The dependence of the slope on redshift has a more robust shape than that of the power spectrum. Therefore, it is simpler to analyze and classify its features. By analyzing the high-redshift minimum of the slope, $z_{\mathrm{dPS} \text {,min }}$, we find that the mean $\operatorname{Ly} \alpha$ intensity at that redshift can be estimated (right panel of Fig. 7). The intensity can be fitted by a simple relation:

$$
\log _{10}\left(J_{\alpha}\right)=a\left(1+z_{\mathrm{dPS}, \min }^{\mathrm{hi}}\right)+b
$$

where $[a, b]=[0.070,-23.1]$. As is evident from the plot, there is a correlation between a model's position in the $z_{\mathrm{dPS}, \min }^{\mathrm{hi}}-J_{\alpha}$ plane and the minimum mass of star-forming halos.

We find a cleaner correlation (Fig. 8) between the redshift of Ly $\alpha$ coupling (i.e., $x_{\alpha}=1$ when averaged over the simulated volume) and $z_{\mathrm{dPS}, \min }^{\mathrm{hi}}$ :

$$
1+z_{\mathrm{Ly} \alpha}=a\left(1+z_{\mathrm{dPS}, \min }^{\mathrm{hi}}\right)+b,
$$

where $[a, b]=[1.08,-5.80]$. We find that in all the models, the Ly $\alpha$ coupling transition happens slightly later than the minimum in the slope (here shown on small scales, where the correlation is clearer).

Cosmic heating can be an extended process starting soon after the first population of luminous sources is formed and extending throughout the first half of reionization. Xrays emitted by the first sources inject their energy into the IGM, driving its temperature up and above that of the CMB. Therefore, properties of the first heating sources are directly related to the thermal evolution of the IGM, which affects the 21-cm signal and its fluctuations. The left panel of Fig. 9 shows how the redshift of the peak heating fluctuations correlates with the mean temperature of the neutral gas in the simulated volume. In other words, with some scatter, the mean gas temperature at high redshifts can be estimated from the position of the heating peak in the $21-\mathrm{cm}$ power spectrum, with the linear fit:

$$
T_{\text {gas }}=a\left(1+z_{\mathrm{PS}, \text { heat }}\right)+b,
$$

where $[a, b]=[1.97,-17.1]$.

The effect of heating fluctuations on the 21-cm signal is most significant around the heating transition $\left(T_{\mathrm{CMB}}=\right.$ $\left.T_{\text {gas }}\right)$ when the other fluctuations vanish. As heating becomes stronger it spatially smooths the signal on small scales, decreasing the slope. Therefore we expect the redshift of the heating transition to correlate with the low-redshift minimum of the slope. Indeed, there is almost a one-to-one correspondence with little scatter, as can be seen in the right panel of Fig. 9. The fitting formula is

$$
\left(1+z_{\mathrm{HT}}\right)=a\left(1+z_{\mathrm{dPS}, \min }^{\mathrm{lo}}\right)+b
$$

where $[a, b]=[0.93,0.70]$. In other words, the low-redshift minimum point is an excellent tracer of the redshift of the heating transition, and can be used to establish the moment at which the IGM was heated to the temperature of the $\mathrm{CMB}$, which of course is known and equals $2.725 \times(1+z) \mathrm{K}$.

The depth of the low-redshift minimum of the slope depends on how efficiently heating acts to smooth small-scale power in the 21-cm signal. X-ray photons with high energies have a large mean free path, thus contributing to smoother heating, and we expect the slope to be low in the scenarios with hard SEDs. On the other hand, if the majority of produced X-rays are soft, heating fluctuations occur on small scales, producing a higher power-spectrum slope. This trend is indeed seen in the simulations. Fig. 10 shows the distribution of the low-redshift minimum point of the slope, with colors indicating the SED of X-ray sources. Ignoring cases with an unreasonably large $\tau$ (marked by crosses), there is the expected clear separation between models with a hard (blue) and soft (red) SED. Models where the X-ray population is mixed and includes both soft sources and miniquasars yield similar predictions to what we find in the cases with a pure soft SED. This is because mini-quasars become significant only at relatively low redshifts. The prediction is clear: if the slope is negative at the low-redshift minimum point, the heating is due to sources emitting mainly hard $\mathrm{X}$-rays; while if it is positive, the heating is very likely dominated by soft X-rays. Thus, measuring the power spectrum at its low-redshift minimum point will allow us to strongly constrain the hardness of X-ray photons and shed light on the nature of their sources. This is complementary to the inference of the X-ray slope of the heating sources that is available based on the anisotropy of the $21-\mathrm{cm}$ power spectrum (Fialkov et al. 2015).

For a generic set of parameters the 21-cm signal during reionization is expected to be strongly affected by both the parameters of heating and the ionizing properties of stars. However, in cases when heating is efficient and saturates early enough, fluctuations in the signal are driven by the bubble structure and density perturbations. As expected, when plotting the neutral fraction at the redshift of the peak versus the peak power during reionization (Fig. 11), we find a one to one correspondence for the models where heating is strong. In these models heating is saturated (or almost saturated) during reionization, which results in a relatively low ionization peak $\left(\Delta_{\mathrm{PS} \text {,ion }}^{2} \lesssim 30 \mathrm{mK}^{2}\right)$. In these cases the 21-cm signal can be directly used as a tracer of the reionization history and as a tool to reconstruct the optical depth to reionization (Barkana 2009; Liu et al. 2016; Fialkov \& Loeb 2016). However, the spectrum does not trace the neutral fraction when heating is weak. In this case the peak is very strong $\left(\Delta_{\mathrm{PS} \text {,ion }}^{2} \gtrsim 150 \mathrm{mK}^{2}\right)$, because the cosmic gas is much colder than the CMB, and the peak occurs when the universe is still mostly neutral; the peak depends strongly on the thermal history in this case. Fig. 11 shows how an ionization peak with a relatively low amplitude $\left(\Delta_{\mathrm{PS} \text {,ion }}^{2} \lesssim 30\right.$ $\mathrm{mK}^{2}$ ) can be used to estimate the neutral fraction at the

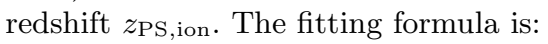

$$
x_{\mathrm{HI}}=a \log _{10}^{2}\left(\Delta_{\mathrm{PS}, \text { ion }}^{2}\right)+b \log _{10}\left(\Delta_{\mathrm{PS}, \text { ion }}^{2}\right)+c,
$$

where $[a, b, c]=[0.047,0.23,0.25]$. On the other hand, most models with low $f_{X}$ have high peaks which are uncorrelated with $x_{\mathrm{HI}}$.

Overall we find that it is difficult to extract useful astrophysical information directly from the features of the power spectrum. In addition, once a peak is detected it might not 

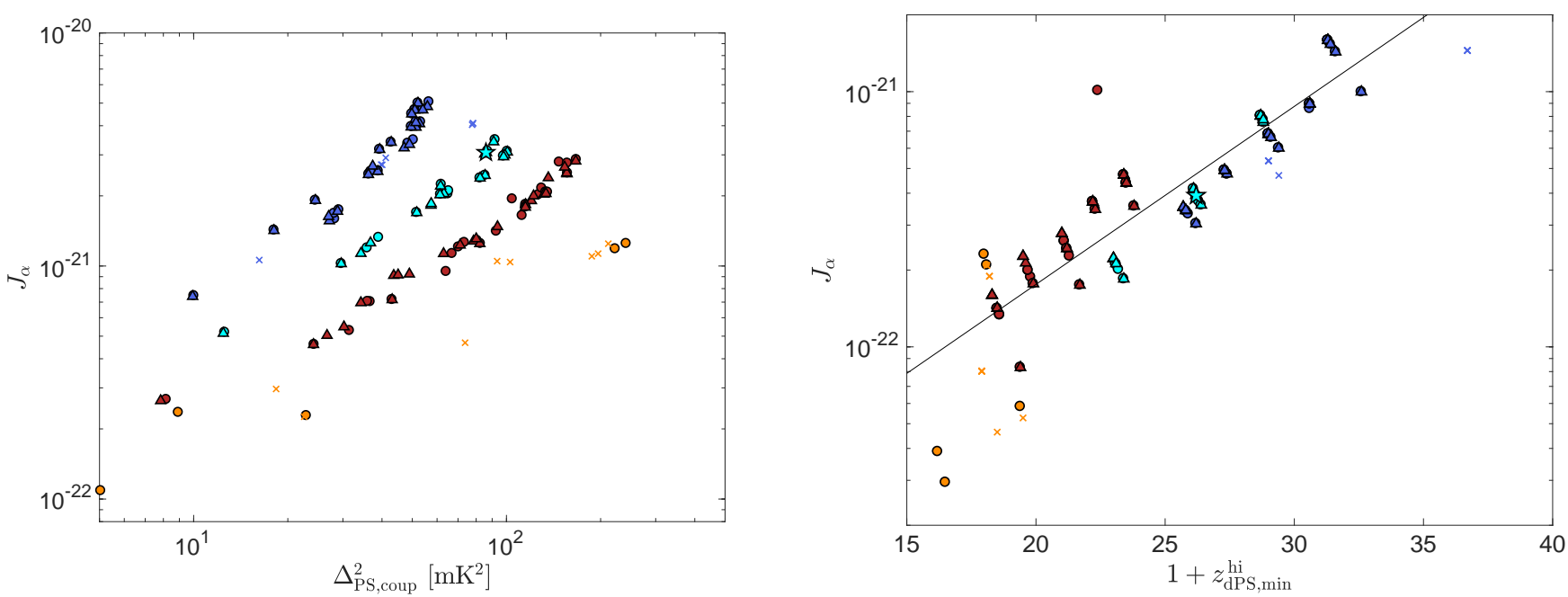

Figure 7. Left: The Ly $\alpha$ intensity in units of $\mathrm{erg} \mathrm{s}^{-1} \mathrm{~cm}^{-2} \mathrm{~Hz}^{-1} \mathrm{sr}^{-1}$ as a function of the power spectrum at $k=0.1 \mathrm{Mpc}-1$ at the Ly $\alpha$ dominant peak. Marker colors indicate the minimum circular velocity of star-forming halos for each case: $V_{c}=4.2$ (blue), 16.5 (cyan), 35.5 (red), and $76.5 \mathrm{~km} \mathrm{~s}^{-1}$ (orange). Marker shapes indicate the optical depth for each case: $\tau=0.060-0.075$ (circles), $0.082-0.09$ (triangles), $0.09-0.111$ (crosses), while the star is our standard case. Right Mean Ly $\alpha$ intensity in units of erg s $\mathrm{cm}^{-2} \mathrm{~Hz}^{-1} \mathrm{sr}^{-1}$ as a function of (one plus) the redshift of the high-redshift minimum of the small-scale power-spectrum slope. Markers (both colors and shapes) are as in the left panel. A solid line shows the best fit, Eq. (6).

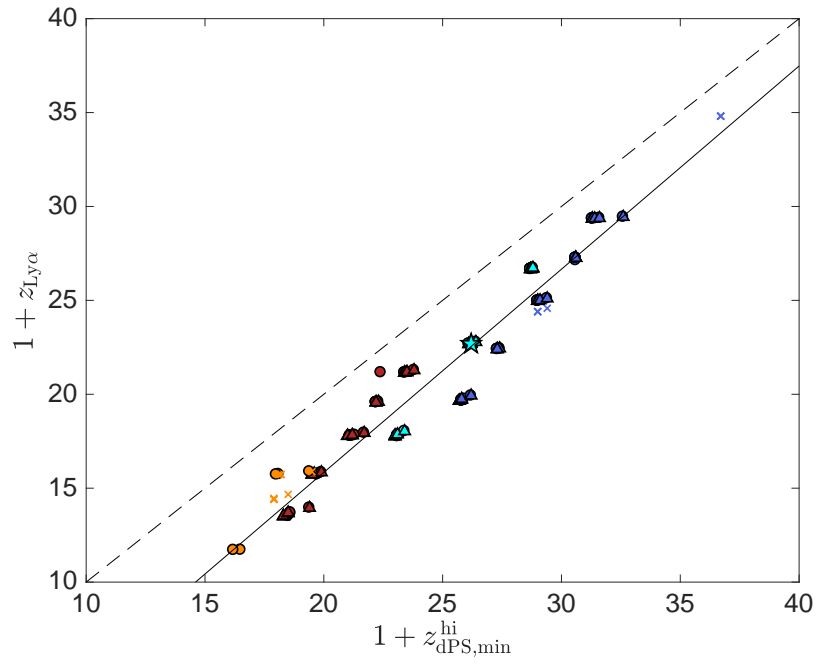

Figure 8. Redshift of the Ly $\alpha$ coupling transition (i.e., $x_{\alpha}=1$ ) as a function of the redshift of the high-redshift minimum of the slope (at $k=0.2-0.6 \mathrm{Mpc}^{-1}$ ). Marker colors indicate the minimum circular velocity of star-forming halos for each case: $V_{c}=4.2$ (blue), 16.5 (cyan), 35.5 (red), and $76.5 \mathrm{~km} \mathrm{~s}^{-1}$ (orange). Marker shapes indicate the optical depth for each case: $\tau=0.060-0.075$ (circles), $0.082-0.09$ (triangles), and $0.09-0.111$ (crosses), while the star is our standard case. We also show the line $Y=X$ (dashed) and the fitting formula from Eq. (7).

always be easy to identify its origin, i.e., whether it is driven by fluctuations in density, coupling, heating or ionization fraction. Out of our 193 cases, 177 have a peak at $k=0.1$ dominated by ionization fluctuations, and it is always the lowest-redshift peak of the power spectrum; the other 16 have a lowest-redshift peak dominated by heating (these are the red circles in the left panel of Fig. 5). The same is true at $k=0.5$ for 155 cases, 34 of which have only one peak, the ionization peak. At $k=0.1,184$ cases have a peak dom- inated by Ly $\alpha$ fluctuations, and it is always the highestredshift peak. At $k=0.1,114$ cases have three power spectrum peaks; the highest-redshift one is Ly $\alpha$-dominated, the lowest-redshift by ionization, and the intermediate one by heating (except for two cases dominated by $\delta+v$ ). We plan to further explore how to classify the peaks directly from observations, using a larger number of simulated cases. The slope of the power spectrum, though, appears to be a more easily useful tool. It is easier to label its features and identify the nature of cosmic events from its redshift evolution.

\section{CONSISTENCY CHECK}

Using our bank of models we can look for relations between the features of the power spectra (or the slope) and the features of the global signal (high-redshift maximum, absorption trough, and emission peak). If such relations exist they could be used as consistency checks to verify the results of observations (e.g., comparing the detected global signal and power spectrum and making sure that there are not any large systematic errors). We do indeed find that the two types of signal are correlated. In this Section we list a few selected relations using the power spectrum slope, rather than directly the power spectrum height, as our main tracer of fluctuations.

The end of the cosmic dark ages, marked by the highredshift maximum in the global signal, is correlated with the high-redshift minimum of the slope, with little scatter (top left panel of Fig. 12). This is because both of these redshifts are directly related to the early Ly $\alpha$ sources. The maximum of the global signal occurs when the Ly $\alpha$ background is first significant, while the minimum of the slope occurs somewhat later, near the Ly $\alpha$ transition. We find the following best-fit relation between the two:

$$
1+z_{\mathrm{g}, \text { max }}^{\mathrm{hi}}=a\left(1+z_{\mathrm{dPS}, \min }^{\mathrm{hi}}\right)+b,
$$



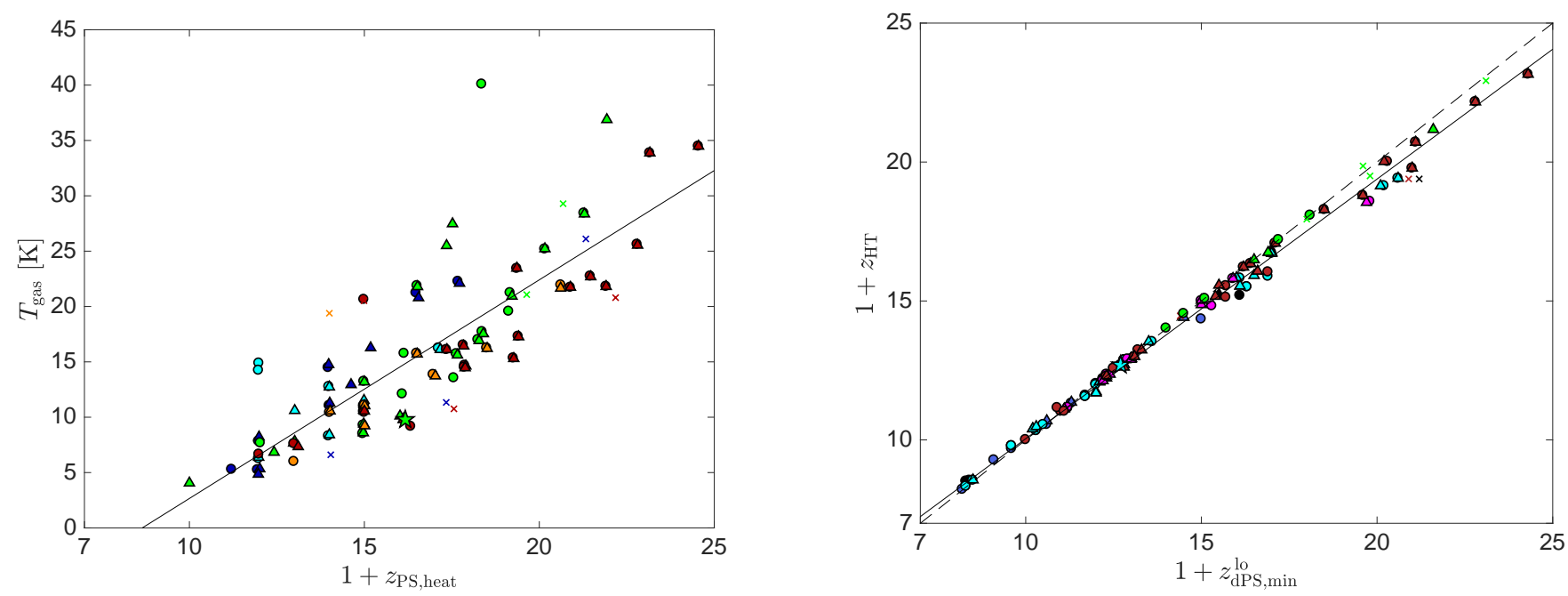

Figure 9. Left: Mean gas temperature (at $z_{\mathrm{PS} \text {,heat }}$ ) as a function of $z_{\mathrm{PS} \text {,heat }}\left(\right.$ at $k=0.1 \mathrm{Mpc}^{-1}$ ). The solid line shows the fitting formula of Eq. (8) . Colors indicate the star formation efficiency for each case: $f_{*}=0.005$ (blue), 0.016 (cyan), 0.05 (green), 0.16 (orange) and 0.5 (red). Shapes indicate the optical depth for each case: $\tau=0.060-0.075$ (circles), $0.082-0.09$ (triangles), $0.09-0.111$ (crosses). Right: Redshift of the heating transition $\left(T_{\mathrm{CMB}}=T_{\text {gas }}\right.$ ) as a function of the redshift of the low-redshift minimum of the slope (at $k=0.2-0.6 \mathrm{Mpc}^{-1}$ ). The colors indicate the star formation efficiency for each case: $f_{X}=0.1$ (black), 0.32 (blue), 1 (cyan), 3.16 (magenta), 8 or 10 (red) and upper limits (green). Shapes indicate the optical depth for each case: $\tau=0.060-0.075$ (circles), $0.082-0.09$ (triangles), $0.09-0.111$ (crosses). Also shown is the $Y=X$ line (dashed) and the fitting formula (solid) of Eq. (9). Note that this plot only includes models that have a heating transition before the end of reionization; the 39 excluded cases can be observationally recognized since they fall in the $\Delta_{\mathrm{PS} \text {,ion }}^{2}>150 \mathrm{mK}^{2}$ part of Fig. 11 (except for one that does not have an ionization peak at $k=0.5 \mathrm{Mpc}^{-1}$ ).

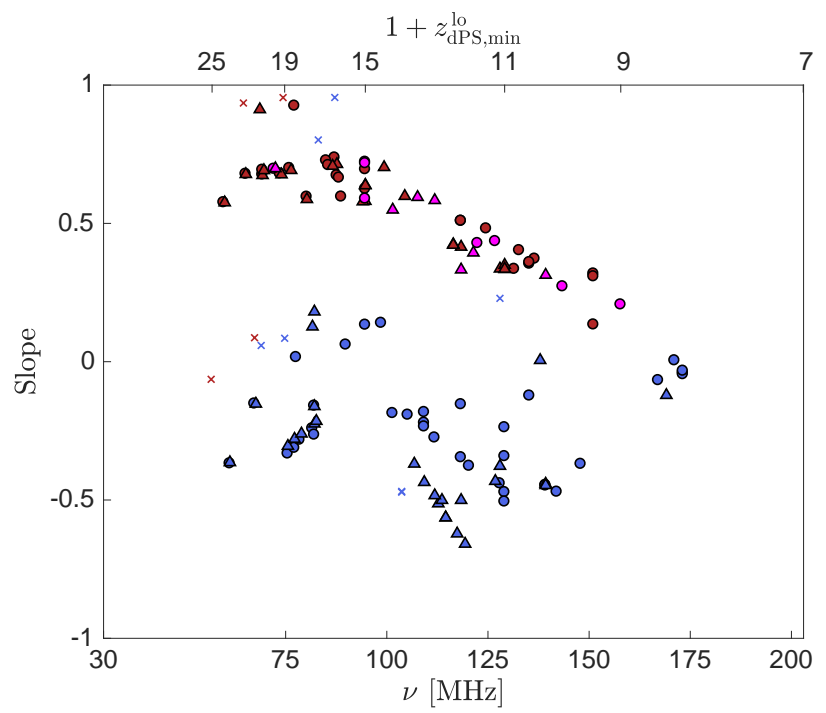

Figure 10. Low-redshift minimum of the large-scale slope (i.e., cyan points from the bottom left panel of Fig. 6), with colors indicating the SED: soft (red), soft \& MQ (magenta) and hard (blue; i.e., XRB, MQ or XRB \& MQ). Shapes indicate the optical depth for each case: $\tau=0.060-0.075$ (circles), $0.082-0.09$ (triangles), $0.09-0.111$ (crosses).

where $[a, b]=[1.14,1.83]$. As we discussed in the previous Section, the redshift of this transition is also related to the minimum mass of halos in which stars form. Therefore, measuring either $z_{\mathrm{g}, \text { max }}^{\mathrm{hi}}$ or $z_{\mathrm{dPS} \text {,min }}^{\mathrm{hi}}$ will yield constraints on the main cooling channel in the early Universe.

The high-redshift maximum of the slope depends on fluctuations in both heating and density. Before the heating transition, these two components have different signs

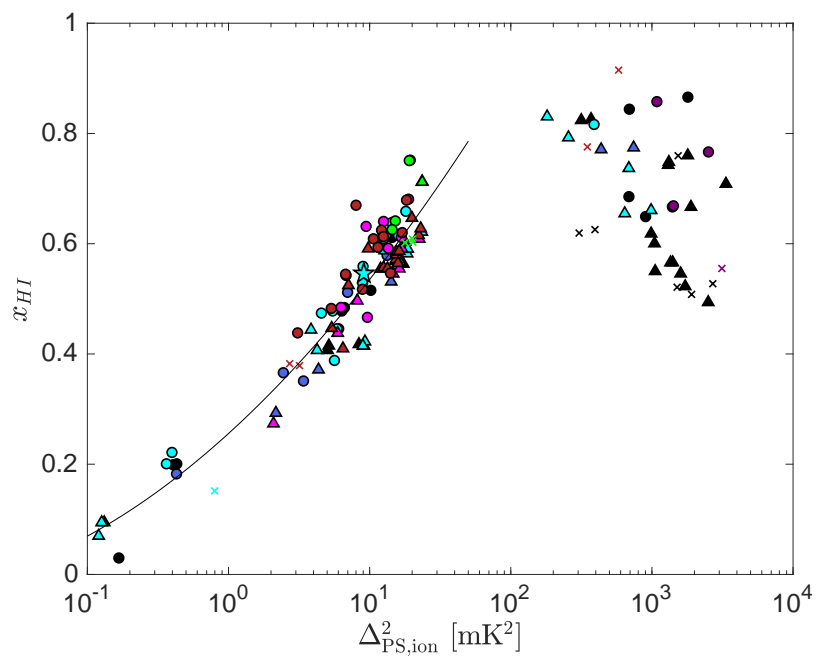

Figure 11. Neutral hydrogen fraction as a function of the power

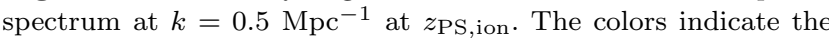
heating efficiency for each case: $f_{X}=0.1$ (black), 0.32 (blue), 1 (cyan), 3.16 (magenta), 8 or 10 (red), lower limits (purple) and upper limits (green). Shapes indicate the optical depth for each case: $\tau=0.060-0.075$ (circles), $0.082-0.09$ (triangles), $0.09-$ 0.111 (crosses). Also shown is the fitting formula from Eq. 10.

(anti-correlate) and partially cancel each other, and at the maximum point the heating fluctuations are large enough to cancel out $\delta+v$ on large scales. We expect this instant to be correlated with the position of the absorption trough of the global signal, $z_{\mathrm{g} \text {,min }}$, which occurs when heating is strong enough to oppose the adiabatic cooling due to the expanding universe and start to heat the gas up. In fact, as we can see from the top right panel of Fig. 12, the two red- 

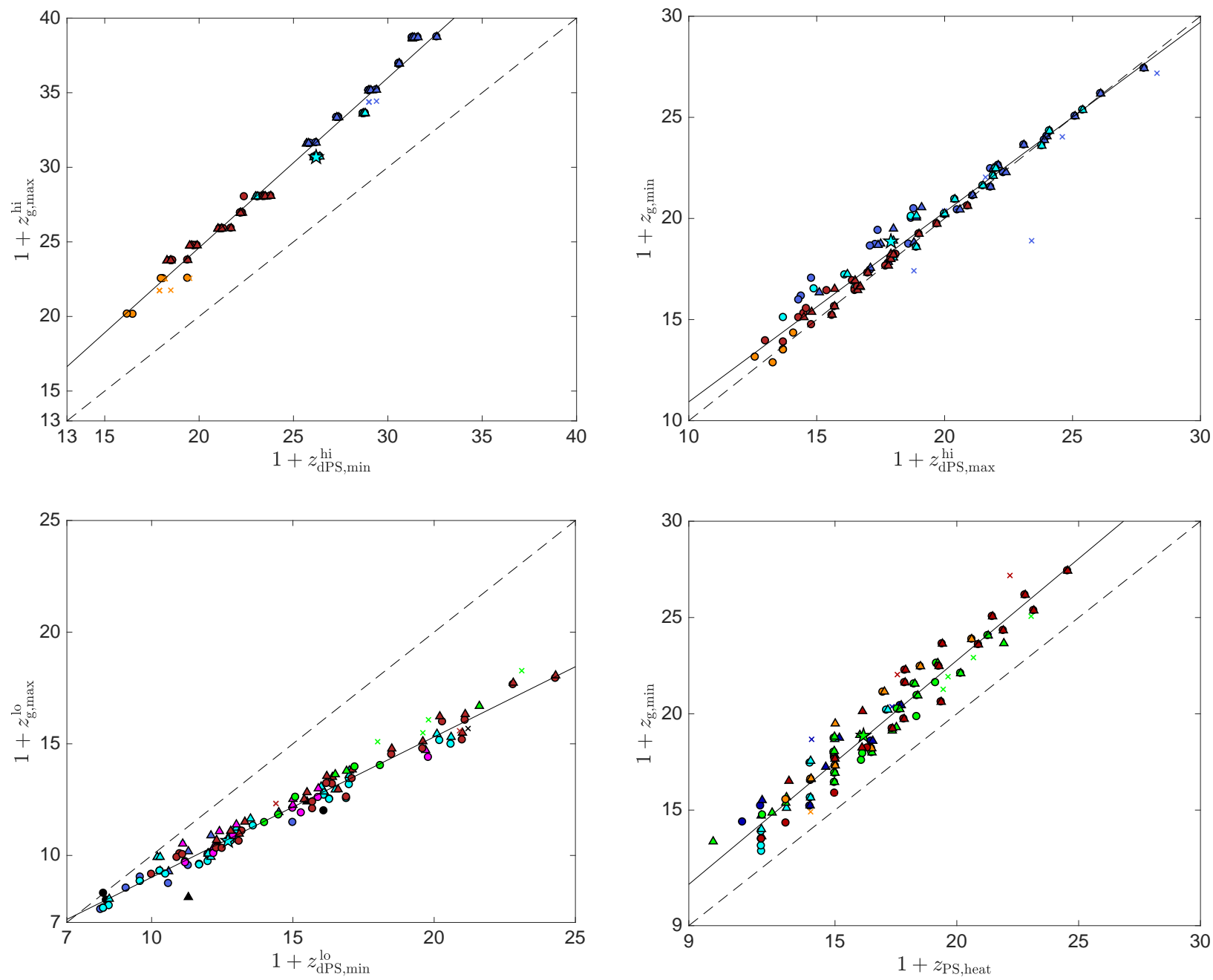

Figure 12. Consistency relations between the features of the global signal and the features of the power spectrum/slope. In addition to the distribution of data points we show the line $Y=X$ (dashed) and a linear fit in each case (solid line). In all panels, marker shapes indicate the optical depth for each case: $\tau=0.060-0.075$ (circles), $0.082-0.09$ (triangles), and $0.09-0.111$ (crosses), while the star is our standard case. Top Left: The redshift of the high-redshift maximum in the global signal as a function of the high-redshift minimum of the small-scale slope $\left(k=0.2-0.6 \mathrm{Mpc}^{-1}\right)$. Marker colors indicate the minimum circular velocity of star-forming halos: $V_{c}=4.2$ (blue), 16.5 (cyan), 35.5 (red), and $76.5 \mathrm{~km} \mathrm{~s}^{-1}$ (orange). We also show the fit, Eq. (11). Top Right: $1+z_{\mathrm{g}, \min }$ versus $1+z_{\mathrm{dPS}, \max }^{\text {hi }}$ (calculated at $k=0.05-0.2 \mathrm{Mpc}^{-1}$ ). Colors and shapes of the markers are as in the left panel. The fitting formula is from Eq. (12). Bottom Left: Redshift of the low-redshift maximum of the global signal as a function of the low-redshift minimum of the slope (at $k=0.2-0.6 \mathrm{Mpc}^{-1}$ ). Marker colors indicate the star formation efficiency for each case: $f_{X}=0.1$ (black), 0.32 (blue), 1 (cyan), 3.16 (magenta), 8 or 10 (red) and upper limits (green). The linear fit is from Eq. (14). Bottom Right: The redshift of the heating peak at

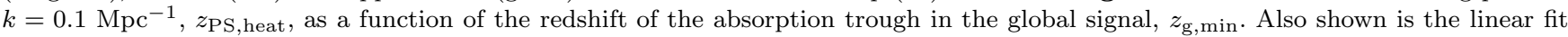
from Eq. (13).

shifts, $z_{\mathrm{g}, \min }$ and $z_{\mathrm{dPS} \text {,max }}^{\mathrm{hi}}$, are nearly equal, with relatively low scatter (especially at high redshifts). In most cases, the minimum point of the global signal occurs first. Our best fit is

$$
1+z_{\mathrm{g}, \min }=a\left(1+z_{\mathrm{dPS}, \max }^{\mathrm{hi}}\right)+b,
$$

where $[a, b]=[0.94,1.55]$. We also show a relation directly with the amplitude of the power spectrum, namely between $z_{\mathrm{g}, \mathrm{min}}$ and the redshift of a peak dominated by heating fluctuations. The correlation, shown on the bottom right panel of Fig. 12, can be fitted by the following formula:

$$
\left(1+z_{\mathrm{g}, \min }\right)=a\left(1+z_{\mathrm{PS}, \text { heat }}\right)+b,
$$

where $[a, b]=[1.06,1.63]$.

Finally, as we know from our earlier work (Figure 7 of Cohen et al. 2016b) both the position and height of the low-redshift maximum of the global signal depend on the average heating rate, as this maximum occurs when heating saturates. Because the redshift of the low-redshift minimum of the slope occurs around the heating transition, we expect $z_{\mathrm{g}, \text { max }}^{\mathrm{hi}}$ and $z_{\mathrm{dPS}, \min }^{\mathrm{lo}}$ to be correlated, with $z_{\mathrm{dPS}, \min }^{\mathrm{lo}}$ somewhat higher. Our models yield the best-fit relation

$$
\left(1+z_{\mathrm{g}, \max }^{\mathrm{hi}}\right)=a\left(1+z_{\mathrm{dPS}, \min }^{\mathrm{lo}}\right)+b,
$$

where $[a, b]=[0.63,2.73]$, shown in the bottom left panel of Fig. 12 


\section{SUMMARY AND DISCUSSION}

The power spectrum of the 21-cm signal contains rich information about the early universe; however, it might be challenging to interpret the signal in terms of astrophysical parameters. In this paper we have used 193 different astrophysical scenarios to build relations between the observable features of the power spectra and astrophysical properties at high redshifts. We applied a semi-numerical simulation to produce a realization of the $21-\mathrm{cm}$ signal for each model, using the same parameter space introduced in detail by Cohen et al. (2016b). The astrophysical parameters can be sub-divided into three main categories: properties of early star formation (minimum halo mass and star formation efficiency), properties of the heating sources (the Xray spectrum and the total luminosity), and properties of reionization (the CMB optical depth). In addition, feedback mechanisms are taken into account when appropriate. The uncertainty in the astrophysical parameters and feedback processes defines wide margins within which the actual 21cm signal can vary. We used the 193 different models to fill up the parameter space and explore possible realizations of the signal.

First, using a few particular cases, we explored the components of the $21-\mathrm{cm}$ signal. There are four possible sources of fluctuations: density (+velocity), Ly $\alpha$ background radiation, heating of the gas in the IGM, and reionization of the IGM. Among these sources, the radiative sources (UV, Xrays and $\operatorname{Ly} \alpha$ ) are usually stressed while the density fluctuations are thought to have a minor contribution at redshifts and scales of interest (accessible by the current and the next generation of ground-based experiments such as the SKA). However, by carefully tagging power spectra peaks and exploring their origins, we found that density fluctuations can play an important role in driving the signal in the intermediate redshift range and on observable scales. This makes the 21-cm signal directly sensitive to fundamental cosmology, though in practice it will still be difficult to separate the density contribution from those of the other sources of 21-cm fluctuations.

The 21-cm power spectrum can have up to three peaks (dominated by density+velocity, Ly $\alpha$, X-rays and/or ionization). After tagging each peak of the power spectrum according to the dominant source of fluctuations, we related the observable properties (redshift and amplitude of the peaks) to the astrophysical inputs in each case. Generally, we found that the slope of the power spectrum (i.e., its dependence on scale) has a more universal structure than the power spectrum. The features include (from high to low redshifts): a minimum point when the Ly $\alpha$ radiative background imprints large scale fluctuations in the 21-cm signal, a maximum when heating cancels density fluctuations on large scales, a minimum when heating dominates, and finally a maximum when ionization cancels out the density and heating fluctuations on large scales. (For cases with inefficient heating, the high-redshift maximum and low-redshift minimum disappear since heating fluctuations are not significant enough.) Moreover, the magnitude of the slope gives useful information for the astrophysical interpretation. A large slope indicates more structure on small scales, and is characteristic of density+velocity or moments where multiple fluctuation sources cancel each other out on large scales.
On the other hand, when a single radiative background dominates, the power spectrum slope is low, indicating that the structure on small scales has been washed out.

We showed results for the cleanest correlations that we found, and provided simple fits. Our main results include:

- A map of the possible locations of peaks (versus redshift) of the power spectrum (Fig. 5 ) and of its slope (bottom panels of Fig. 6).

- The redshift of the Ly $\alpha$ coupling transition can be estimated from the redshift of the high-redshift minimum of the slope (Fig. 8).

- The redshift of the heating transition (and the mean gas temperature of the IGM at that time) can be estimated from the redshift of the low-redshift minimum of the slope (right panel of Fig. 9).

- The spectrum of the X-ray heating sources can be estimated from the properties of the low-redshift minimum of the slope (Fig. 10).

- By measuring the properties of the ionizationdominated peak of the power spectrum, it is possible to deduce whether heating occurred early or late; and if early, then the mean reionized fraction of the IGM at that time can also be estimated (Fig. 11).

- Significant correlations (with low scatter) are expected between some peaks of the power spectrum and its slope, and peaks of the global 21-cm signal (Fig. 12). This will give an important consistency check once these various observations are made.

\section{ACKNOWLEDGMENTS}

This project/publication was made possible through the support of a grant from the John Templeton Foundation. The opinions expressed in this publication are those of the authors and do not necessarily reflect the views of the John Templeton Foundation. R.B. and A.C. also acknowledge Israel Science Foundation grant 823/09 and the Ministry of Science and Technology, Israel. R.B.'s work has been partly done within the Labex Institut Lagrange de Paris (ILP, reference ANR-10-LABX-63) part of the Idex SUPER, and received financial state aid managed by the Agence Nationale de la Recherche, as part of the programme Investissements d'avenir under the reference ANR-11-IDEX-0004-02. R.B. also acknowledges a Leverhulme Trust Visiting Professorship. This research was supported in part by Perimeter Institute for Theoretical Physics. Research at Perimeter Institute is supported by the Government of Canada through the Department of Innovation, Science and Economic Development Canada and by the Province of Ontario through the Ministry of Research, Innovation and Science.

\section{REFERENCES}

Ali, Z. S., et al., 2015, ApJ, 809, 61

Barkana, R., 2008, MNRAS, 391, 727

Barkana, R. 2009, MNRAS, 397, 1454

Barkana, R., 2016, Physics Reports, 645, 1

Barkana, R., \& Loeb, A., 2001, Phys. Rep., 349, 125

Barkana, R., \& Loeb, A. 2004, ApJ, 609, 474

Barkana, R., \& Loeb, A. 2005a, ApJ, 624, L65 
Barkana, R., \& Loeb, A. 2005b, ApJ, 626, 1

Beardsley A. P., et al., 2016, ApJ, 833, 102

Bernardi, G., Zwart, J. T. L., Price, D., Greenhill, L. J.,

Mesinger, A., et al., 2016, MNRAS, 461, 2847

Bharadwaj, S., \& Ali, S. S. 2004, MNRAS, 352, 142

Bowman, J. D. et al., 2013, PASA, 30, 31

Bowman, J. D., \& Rogers, A. E. E., 2010, Nature, 468, 796

Bromm V., Coppi P. S., \& Larson R. B., 2002, ApJ, 564, 23

Brorby, M., Kaaret, P., Prestwich, A., Mirabel, I. F., 2016, MNRAS, 457, 4081

Burns, J., Lazio, J., Bowman, J., Bradley, R., Datta, A., et al., 2015, IAUGA, 2229646

Chen, X., Miralda-Escudé, J., 2004, ApJ, 602, 1

Chuzhoy, L., \& Shapiro, P. R., 2006, ApJ, 651, 1

Cohen, A., Fialkov, A., \& Barkana, R., 2016a, MNRAS, 459, L90

Cohen, A., Fialkov, A., Barkana, R., \& Lotem, M., 2016b, arXiv:1609.02312

Dalal, N., Pen, U.-L., \& Seljak, U. 2010, JCAP, 11, 007

DeBoer, D. R., Parsons, A. R., Aguirre, J. E., et al., 2016, arXiv:1606.07473

Dijkstra, M., Gilfanov, M., Loeb, A., Sunyaev, R., 2012, MNRAS, 421, 213

Fialkov, A., Barkana, R., Tseliakhovich, D., Hirata, C., 2012, MNRAS, 424, 1335

Fialkov, A., Barkana, R., Visbal, E., Tseliakhovich, D., Hirata, C., 2013, MNRAS, 432, 2909

Fialkov, A., 2014, IJMPD, 2330017

Fialkov, A., Barkana, R., \& Visbal, E., 2014, Nature, 506, 197

Fialkov, A., \& Barkana, R., 2014, MNRAS, 445, 213

Fialkov, A., Barkana, R., Pinhas, A., \& Visbal, E., 2014, MNRAS, 437, 36

Fialkov, A., Barkana, R., \& Cohen, A. 2015, Physical Review Letters, 114, 101303

Fialkov, A., Loeb, A., 2016, ApJ, 821, 59

Fialkov, A., Cohen, A., Barkana, R., \& Silk, J., 2017, MNRAS, 464, 3498

Field, G. B., 1958, Proc. IRE 46, 240

Fragos, T., Lehmer, B. D., Naoz, S., Zezas, A., \& BasuZych, A., 2013, ApJ, 776, L31

Furlanetto, S. R., 2006, MNRAS 371, 867

Furlanetto, S. R., Oh, S. P., \& Briggs, F. H., 2006, PhR 433, 181

Greig, B., \& Mesinger, A. 2015, MNRAS, 449, 4246

Gunn, J. E., \& Peterson, B. A., 1965, ApJ, 142, 1633

Haiman, Z., Rees, M. J., Loeb, A., 1997, ApJ, 476, 458

Haiman, Z., Abel, T. \& Rees, M. J., 2000, ApJ, 534, 11

Hassan, S., Dave, R., Finlator, K., \& Santos, M. G. 2016, arXiv:1612.05797

Hirata, C. M., 2006, MNRAS, 367, 259

Jacobs D. C., et al., 2015, The Astrophysical Journal, 801, 51

Jeon, M., Pawlik, A. H., Bromm, V., Milosavljevic, M., 2014, MNRAS, 444, 3288

Koopmans, L., Pritchard, J., Mellema, G., Aguirre, J., Ahn, K., et al. 2015, Proceedings of Advancing Astrophysics with the Square Kilometre Array (AASKA14). 9 -13 June, 2014. Giardini Naxos, Italy

Lehmer, B. D., Xue, Y. Q., Brandt, W. N., Alexander, D. M., Bauer, F. E., et al., 2012, ApJ, 752, 46L
Liu, A., Pritchard, J., Allison, R., Parsons, A. R., Seljak, U., Sherwin, B. D., 2016, PRD, 93, 3013

Machacek, M. E., Bryan, G. L., Abel, T., 2001, ApJ, 548, 509

Madau, P., Rees, M. J., Volonteri, M., Haardt, F., \& Oh, S. P., 2004, ApJ, 604, 484

Madau, P. \& Haardt, F., 2015, ApJ, 813L, 8

Madau, P. \& Fragos, T., arXiv:160607887

Mesinger, A., Furlanetto, S., Cen, R. 2011, MNRAS, 411, 955

Mirabel, I. F., Dijkstra, M., Laurent, P., Loeb, A., \& Pritchard, J. R., 2011, A\&A, 528, 149

Mirocha, Furlanetto, S. R. \& Sun, G., 2016, arXiv:1607.00386

Monsalve, R. A., Rogers, A. E. E., Bowman, J. D., Mozdzen, T. J., 2017, ApJ, 835, 49

Navarro, J. F., Steinmetz, M. 2000, ApJ, 538, 477

O'Shea, B. W. \& Norman, M. L., 2008, ApJ, 673, 14

O'Shea, B. W., Wise, J. H., Xu, H., Norman, M. L., 2015, ApJ, 807, 12

Pacucci, F., Mesinger, A., Mineo, S., \& Ferrara, A. 2014, MNRAS, 443, 678

Parsons A. R., et al., 2014, ApJ, 788, 106

Patil A. H., et al., 2017, ApJ, 838, 65

Planck Collaboration, Aghanim, N., Ashdown, M., et al. 2016a, A\&A, 596, A107

Planck Collaboration, Adam, R., Aghanim, N., et al. 2016b, A\&A, 596, A108

Planck Collaboration, Ade, P. A. R., Aghanim, N., et al. 2016b, A\&A, 594, A13

Pober, J. C., et al. 2014, ApJ, 782, 66

Pober, J. C., Ali, Z. S., Parsons, A. R., et al., 2015, ApJ, 809, 62

Pritchard, J. R., Furlanetto, S. R., 2006, MNRAS, 372, 1093

Pritchard, J. R., Furlanetto, S. R. 2007, MNRAS, 376, 1680

Pritchard, J. R., Loeb, A., 2008, PRD, 78, 3511

Rees, M. J. 1986, MNRAS, 222, 27

Schauer, A. T. P., Whalen, D. J., Glover, S. C. O., Klessen, R. S., 2015, MNRAS, 454, 2441

Shimabukuro, H., \& Semelin, B. 2017, arXiv:1701.07026

Singh, S., Subrahmanyan, R., Udaya Shankar, N., et al. 2017, arXiv:1703.06647

Sobacchi, E., \& Mesinger, A., 2013, MNRAS, 432, 3340

Tanaka, T., Perna, R., \& Haiman, Z.,2012, MNRAS, 425, 2974

Tegmark, M., Silk, J., Rees, M., Blanchard, A., Abel, T., \& Palla, F., 1997, ApJ, 474, 1

Tseliakhovich, D., \& Hirata, C. M., 2010, PRD, 82, 3520

Tseliakhovich D., Barkana R., \& Hirata C. M. 2011, MNRAS, 418, 906

van Haarlem, M. P. et al., 2013, A\&A, 556, 2.

Visbal, E., Barkana, R., Fialkov, A., Tseliakhovich, D., Hirata, C. M., 2012, Nature, 487, 70

Visbal, E., Zoltan, H., Terrazas, B., Bryan, G. L., Barkana, R., 2014, MNRAS, 445, 107

Weinberg, D. H., Hernquist, L., Katz, N. 1997, ApJ, 477, 8

Wise, J. H. \& Abel, T., 2007, ApJ, 671, 1559

Wise, J. H., Demchenko, V. G., Halicek, M. T., Norman, M. L., Turk, M. J., Abel, T., \& Smith, B. D., 2014, MNRAS, 442,2560 
Wouthuysen, S. A., 1952, Astron. J. 57, 31

Wyithe, J. S. B., \& Loeb, A., 2013, MNRAS, 428, 2741

Xu, H., Wise, J. H., Norman, M. L., Ahn, K., \& O'Shea, B. W., 2016, ApJ, 833, 84

Yoshida N., Abel T., Hernquist L., \& Sugiyama N., 2003, ApJ, 592, 645

Zarka, P., Girard, J. N., Tagger, M., \& Denis, L. 2012, SF2A-2012: Proceedings of the Annual meeting of the French Society of Astronomy and Astrophysics

\section{APPENDIX A: CASES LIST \\ APPENDIX B: ADDITIONAL FIGURES}

To demonstrate the variety of possible realizations of the power spectra and corresponding slopes we show figures analogues to Fig. 1 and 3 but for various sets of astrophysical parameters.

First we show variations of our standard case, altering one astrophysical parameter at a time. The left column of Fig. B1 presents the case of strong X-rays with $f_{X}=8$ instead of $f_{X}=1$ (\#57 in Table A1). Because of the stronger heating, the Ly $\alpha$ peak becomes narrower compared to the standard case. This is because in model \# 57 heating starts earlier and X-rays (which anti-correlate with Ly $\alpha$ ) cut the Ly $\alpha$ peak off. For the same reason, the features of the slope (high- $z \max$ and low- $z$ min) shift to higher redshifts. Compared to the standard case, the heating peak in this case is more prominent and occurs earlier, but is still relatively small as expected with hard X-rays. Next, we show a case with more massive halos (\#88, right column of Fig. B1), setting the minimum $V_{c}=35.5 \mathrm{~km} \mathrm{~s}^{-1}$ instead of $V_{c}=16.5$ $\mathrm{km} \mathrm{s}^{-1}$ in the standard case. In this case all the cosmological milestones are shifted to lower redshifts, as heavier halos can only form stars somewhat later (though the timing of reionization is fixed since we have not changed $\tau$ ). The fluctuations are higher in this case because of the higher bias of the more massive galactic halos, and reionization occurs over a smaller redshift interval.

The left column of Fig. B2 shows a case with a low SFR $\left(f_{*}=0.005, \# 41\right.$, compared to $f_{*}=0.05$ in the standard case). In this case, because of the inefficient star formation, it takes longer to build up the radiative backgrounds. Halo formation advances more rapidly at higher redshifts, so while Ly $\alpha$ coupling is delayed, cosmic heating is delayed far more. As a result, the power spectrum peaks are wider. Moreover, in this case density fluctuations dominate the high-redshift peak on both large and small scales. The right column of Fig. B2 shows an unusual case (\#108) in which the fluctuation level remains consistenly high over a wide range of high redshifts. This case has the earliest possible high star formation, in that the star formation efficiency is very high $\left(f_{*}=50 \%\right)$ and stars are assumed to form in the lowest possible halo masses (corresponding to the molecular cooling threshold). This leads to an early saturation of Ly $\alpha$ coupling. However, this model assumes mini-quasars as the only $\mathrm{X}$-ray heating source, so heating occurs relatively late. As a result, the Ly $\alpha$ and heating era are well separated in time allowing density fluctuations to dominate the signal at intermediate redshifts (where the global signal shows strong absorption and thus the fluctuations are strong as well).
Note, though, that this case has an optical depth of 0.098 to the CMB, which is ruled out at about the $3-\sigma$ level (Planck Collaboration et al. 2016b).

Fig. B3 shows two examples of cases with slopes that have unusual structure (i.e., different from the typical shape of two minima and two maxima). The left panel presents a case (\#37) with very low efficiencies of SFR and heating ( $f_{*}=0.005$ and $f_{X}=0.1$ ), so that there is no heating transition before the end of reionization. As a result, the highredshift maximum and low-redshift minimum of the slope disappear. Note that in this type of case, the low-redshift maximum occurs when ionization and heating fluctuations together cancel out density+velocity on large scales. Finally, we show a case (right panel of Fig. B3. \#122) with very weak star formation (as it is limited to very massive halos, with $V_{c}=76.5 \mathrm{~km} / \mathrm{s}$, and even there it has a low efficiency). This case has an extra bump (i.e., small maximum) at very high redshifts due to late $\operatorname{Ly} \alpha$ coupling. 


\begin{tabular}{|c|c|c|c|c|c|c|c|c|c|c|}
\hline$\#$ & & $f_{*}$ & $V_{c}[\mathrm{~km} / \mathrm{s}]$ & $f_{X}$ & SED & $\tau$ & LW & Low-mass cutoff & $\zeta$ & $R_{\mathrm{mfp}}[\mathrm{Mpc}]$ \\
\hline 1 & Filler & 0.005 & 4.2 & 0.1 & Hard & 0.066 & On & Eq. (4) from C2016b & 20 & 70 \\
\hline 2 & Filler & 0.005 & 4.2 & 0.1 & Hard & 0.082 & On & Eq. (4) from C2016b & 32 & 70 \\
\hline 3 & Filler & 0.005 & 4.2 & 0.1 & Soft & 0.066 & On & Eq. (4) from C2016b & 20 & 70 \\
\hline 4 & Filler & 0.005 & 4.2 & 0.1 & Soft & 0.082 & On & Eq. (4) from C2016b & 32 & 70 \\
\hline 5 & Filler & 0.005 & 4.2 & 1 & Hard & 0.066 & On & Eq. (4) from C2016b & 20 & 70 \\
\hline 6 & Filler & 0.005 & 4.2 & 1 & Hard & 0.082 & On & Eq. (4) from C2016b & 32 & 70 \\
\hline 7 & Filler & 0.005 & 4.2 & 1 & Soft & 0.066 & On & Eq. (4) from C2016b & 20 & 70 \\
\hline 8 & Filler & 0.005 & 4.2 & 1 & Soft & 0.082 & On & Eq. (4) from C2016b & 32 & 70 \\
\hline 9 & Filler & 0.005 & 4.2 & 8 & Hard & 0.066 & On & Eq. (4) from C2016b & 20 & 70 \\
\hline 10 & Filler & 0.005 & 4.2 & 8 & Hard & 0.082 & On & Eq. (4) from C2016b & 32 & 70 \\
\hline 11 & Filler & 0.005 & 4.2 & 8 & Soft & 0.066 & On & Eq. (4) from C2016b & 19 & 70 \\
\hline 12 & Filler & 0.005 & 4.2 & 8 & Soft & 0.082 & On & Eq. (4) from C2016b & 31 & 70 \\
\hline 13 & Filler & 0.05 & 4.2 & 0.1 & Hard & 0.066 & On & Eq. (4) from C2016b & 20 & 70 \\
\hline 14 & Filler & 0.05 & 4.2 & 0.1 & Hard & 0.082 & On & Eq. (4) from C2016b & 36 & 70 \\
\hline 15 & Filler & 0.05 & 4.2 & 0.1 & Soft & 0.066 & On & Eq. (4) from C2016b & 20 & 70 \\
\hline 16 & Filler & 0.05 & 4.2 & 0.1 & Soft & 0.082 & On & Eq. (4) from C2016b & 36 & 70 \\
\hline 17 & Filler & 0.05 & 4.2 & 1 & Hard & 0.066 & On & Eq. (4) from C2016b & 20 & 70 \\
\hline 18 & Filler & 0.05 & 4.2 & 1 & Hard & 0.082 & On & Eq. (4) from C2016b & 36 & 70 \\
\hline 19 & Filler & 0.05 & 4.2 & 1 & Soft & 0.066 & On & Eq. (4) from C2016b & 19 & 70 \\
\hline 20 & Filler & 0.05 & 4.2 & 1 & Soft & 0.082 & On & Eq. (4) from C2016b & 35 & 70 \\
\hline 21 & Filler & 0.05 & 4.2 & 8 & Hard & 0.066 & On & Eq. (4) from C2016b & 18 & 70 \\
\hline 22 & Filler & 0.05 & 4.2 & 8 & Hard & 0.082 & On & Eq. (4) from C2016b & 34 & 70 \\
\hline 23 & Filler & 0.05 & 4.2 & 8 & Soft & 0.066 & On & Eq. (4) from C2016b & 15 & 70 \\
\hline 24 & Filler & 0.05 & 4.2 & 8 & Soft & 0.082 & On & Eq. (4) from C2016b & 31 & 70 \\
\hline 25 & Filler & 0.5 & 4.2 & 0.1 & Hard & 0.066 & On & Eq. (4) from C2016b & 26 & 70 \\
\hline 26 & Filler & 0.5 & 4.2 & 0.1 & Hard & 0.082 & On & Eq. (4) from C2016b & 51 & 70 \\
\hline 27 & Filler & 0.5 & 4.2 & 0.1 & Soft & 0.066 & On & Eq. (4) from C2016b & 25 & 70 \\
\hline 28 & Filler & 0.5 & 4.2 & 0.1 & Soft & 0.082 & On & Eq. (4) from C2016b & 51 & 70 \\
\hline 29 & Filler & 0.5 & 4.2 & 1 & Hard & 0.066 & On & Eq. (4) from C2016b & 24 & 70 \\
\hline 30 & Filler & 0.5 & 4.2 & 1 & Hard & 0.082 & On & Eq. (4) from C2016b & 50 & 70 \\
\hline 31 & Filler & 0.5 & 4.2 & 1 & Soft & 0.066 & On & Eq. (4) from C2016b & 20 & 70 \\
\hline 32 & Filler & 0.5 & 4.2 & 1 & Soft & 0.082 & On & Eq. (4) from C2016b & 44 & 70 \\
\hline 33 & Filler & 0.5 & 4.2 & 8 & Hard & 0.066 & On & Eq. (4) from C2016b & 16 & 70 \\
\hline 34 & Filler & 0.5 & 4.2 & 8 & Hard & 0.082 & On & Eq. (4) from C2016b & 39 & 70 \\
\hline 35 & Filler & 0.5 & 4.2 & 8 & Soft & 0.066 & On & Eq. (4) from C2016b & 1.5 & 70 \\
\hline 36 & Filler & 0.5 & 4.2 & 8 & Soft & 0.082 & On & Eq. (4) from C2016b & 19 & 70 \\
\hline 37 & Filler & 0.005 & 16.5 & 0.1 & Hard & 0.066 & - & - & 20 & 70 \\
\hline 38 & Filler & 0.005 & 16.5 & 0.1 & Hard & 0.082 & - & - & 37 & 70 \\
\hline 39 & Filler & 0.005 & 16.5 & 0.1 & Soft & 0.066 & - & - & 20 & 70 \\
\hline 40 & Filler & 0.005 & 16.5 & 0.1 & Soft & 0.082 & - & - & 37 & 70 \\
\hline 41 & Filler & 0.005 & 16.5 & 1 & Hard & 0.066 & - & - & 20 & 70 \\
\hline 42 & Filler & 0.005 & 16.5 & 1 & Hard & 0.082 & - & - & 37 & 70 \\
\hline 43 & Filler & 0.005 & 16.5 & 1 & Soft & 0.066 & - & - & 20 & 70 \\
\hline 44 & Filler & 0.005 & 16.5 & 1 & Soft & 0.082 & - & - & 36 & 70 \\
\hline 45 & Filler & 0.005 & 16.5 & 8 & Hard & 0.066 & - & - & 20 & 70 \\
\hline 46 & Filler & 0.005 & 16.5 & 8 & Hard & 0.082 & - & - & 36 & 70 \\
\hline 47 & Filler & 0.005 & 16.5 & 8 & Soft & 0.066 & - & - & 19 & 70 \\
\hline 48 & Filler & 0.005 & 16.5 & 8 & Soft & 0.082 & - & - & 36 & 70 \\
\hline 49 & Filler & 0.05 & 16.5 & 0.1 & Hard & 0.066 & - & - & 20 & 70 \\
\hline 50 & Filler & 0.05 & 16.5 & 0.1 & Hard & 0.082 & - & - & 37 & 70 \\
\hline 51 & Filler & 0.05 & 16.5 & 0.1 & Soft & 0.066 & - & - & 20 & 70 \\
\hline 52 & Filler & 0.05 & 16.5 & 0.1 & Soft & 0.082 & - & - & 36 & 70 \\
\hline 53 & Standard & 0.05 & 16.5 & 1 & Hard & 0.066 & - & - & 20 & 70 \\
\hline 54 & Filler & 0.05 & 16.5 & 1 & Hard & 0.082 & - & - & 36 & 70 \\
\hline 55 & Filler & 0.05 & 16.5 & 1 & Soft & 0.066 & - & - & 19 & 70 \\
\hline 56 & Filler & 0.05 & 16.5 & 1 & Soft & 0.082 & - & - & 36 & 70 \\
\hline 57 & Filler & 0.05 & 16.5 & 8 & Hard & 0.066 & - & - & 19 & 70 \\
\hline 58 & Filler & 0.05 & 16.5 & 8 & Hard & 0.082 & - & - & 35 & 70 \\
\hline 59 & Filler & 0.05 & 16.5 & 8 & Soft & 0.066 & - & - & 15 & 70 \\
\hline 60 & Filler & 0.05 & 16.5 & 8 & Soft & 0.082 & - & - & 31 & 70 \\
\hline 61 & Filler & 0.5 & 16.5 & 0.1 & Hard & 0.066 & - & - & 20 & 70 \\
\hline
\end{tabular}




\begin{tabular}{|c|c|c|c|c|c|c|c|c|c|c|}
\hline$\#$ & & $f_{*}$ & $V_{c}[\mathrm{~km} / \mathrm{s}]$ & $f_{X}$ & SED & $\tau$ & LW & Low-mass cutoff & $\zeta$ & $R_{\mathrm{mfp}}[\mathrm{Mpc}]$ \\
\hline 62 & Filler & 0.5 & 16.5 & 0.1 & Hard & 0.082 & - & - & 36 & 70 \\
\hline 63 & Filler & 0.5 & 16.5 & 0.1 & Soft & 0.066 & - & - & 19 & 70 \\
\hline 64 & Filler & 0.5 & 16.5 & 0.1 & Soft & 0.082 & - & - & 36 & 70 \\
\hline 65 & Filler & 0.5 & 16.5 & 1 & Hard & 0.066 & - & - & 18 & 70 \\
\hline 66 & Filler & 0.5 & 16.5 & 1 & Hard & 0.082 & - & - & 35 & 70 \\
\hline 67 & Filler & 0.5 & 16.5 & 1 & Soft & 0.066 & - & - & 14 & 70 \\
\hline 68 & Filler & 0.5 & 16.5 & 1 & Soft & 0.082 & - & - & 30 & 70 \\
\hline 69 & Filler & 0.5 & 16.5 & 8 & Hard & 0.066 & - & - & 11.5 & 70 \\
\hline 70 & Filler & 0.5 & 16.5 & 8 & Hard & 0.082 & - & - & 27 & 70 \\
\hline 71 & Filler & 0.5 & 16.5 & 8 & Soft & 0.066 & - & - & 0.01 & 70 \\
\hline 72 & Filler & 0.5 & 16.5 & 8 & Soft & 0.082 & - & - & 9 & 70 \\
\hline 73 & Filler & 0.005 & 35.5 & 0.1 & Hard & 0.082 & - & - & 130 & 70 \\
\hline 74 & Filler & 0.005 & 35.5 & 0.1 & Soft & 0.066 & - & - & 53 & 70 \\
\hline 75 & Filler & 0.005 & 35.5 & 0.1 & Soft & 0.082 & - & - & 130 & 70 \\
\hline 76 & Filler & 0.005 & 35.5 & 1 & Hard & 0.066 & - & - & 53 & 70 \\
\hline 77 & Filler & 0.005 & 35.5 & 1 & Hard & 0.082 & - & - & 130 & 70 \\
\hline 78 & Filler & 0.005 & 35.5 & 1 & Soft & 0.066 & - & - & 53 & 70 \\
\hline 79 & Filler & 0.005 & 35.5 & 1 & Soft & 0.082 & - & - & 130 & 70 \\
\hline 80 & Filler & 0.005 & 35.5 & 8 & Hard & 0.066 & - & - & 53 & 70 \\
\hline 81 & Filler & 0.005 & 35.5 & 8 & Hard & 0.082 & - & - & 130 & 70 \\
\hline 82 & Filler & 0.005 & 35.5 & 8 & Soft & 0.066 & - & - & 53 & 70 \\
\hline 83 & Filler & 0.005 & 35.5 & 8 & Soft & 0.082 & - & - & 129 & 70 \\
\hline 84 & Filler & 0.05 & 35.5 & 0.1 & Hard & 0.066 & - & - & 53 & 70 \\
\hline 85 & Filler & 0.05 & 35.5 & 0.1 & Hard & 0.082 & - & - & 130 & 70 \\
\hline 86 & Filler & 0.05 & 35.5 & 0.1 & Soft & 0.066 & - & - & 53 & 70 \\
\hline 87 & Filler & 0.05 & 35.5 & 0.1 & Soft & 0.082 & - & - & 130 & 70 \\
\hline 88 & Filler & 0.05 & 35.5 & 1 & Hard & 0.066 & - & - & 53 & 70 \\
\hline 89 & Filler & 0.05 & 35.5 & 1 & Hard & 0.082 & - & - & 130 & 70 \\
\hline 90 & Filler & 0.05 & 35.5 & 1 & Soft & 0.066 & - & - & 52 & 70 \\
\hline 91 & Filler & 0.05 & 35.5 & 1 & Soft & 0.082 & - & - & 129 & 70 \\
\hline 92 & Filler & 0.05 & 35.5 & 8 & Hard & 0.066 & - & - & 52 & 70 \\
\hline 93 & Filler & 0.05 & 35.5 & 8 & Hard & 0.082 & - & - & 129 & 70 \\
\hline 94 & Filler & 0.05 & 35.5 & 8 & Soft & 0.066 & - & - & 48 & 70 \\
\hline 95 & Filler & 0.05 & 35.5 & 8 & Soft & 0.082 & - & - & 124 & 70 \\
\hline 96 & Filler & 0.5 & 35.5 & 0.1 & Hard & 0.066 & - & - & 53 & 70 \\
\hline 97 & Filler & 0.5 & 35.5 & 0.1 & Hard & 0.082 & - & - & 130 & 70 \\
\hline 98 & Filler & 0.5 & 35.5 & 0.1 & Soft & 0.066 & - & - & 52 & 70 \\
\hline 99 & Filler & 0.5 & 35.5 & 0.1 & Soft & 0.082 & - & - & 129 & 70 \\
\hline 100 & Filler & 0.5 & 35.5 & 1 & Hard & 0.066 & - & - & 52 & 70 \\
\hline 101 & Filler & 0.5 & 35.5 & 1 & Hard & 0.082 & - & - & 129 & 70 \\
\hline 102 & Filler & 0.5 & 35.5 & 1 & Soft & 0.066 & - & - & 47 & 70 \\
\hline 103 & Filler & 0.5 & 35.5 & 1 & Soft & 0.082 & - & - & 122 & 70 \\
\hline 104 & Filler & 0.5 & 35.5 & 8 & Hard & 0.066 & - & - & 43 & 70 \\
\hline 105 & Filler & 0.5 & 35.5 & 8 & Hard & 0.082 & - & - & 119 & 70 \\
\hline 106 & Filler & 0.5 & 35.5 & 8 & Soft & 0.066 & - & - & 22 & 70 \\
\hline 107 & Filler & 0.5 & 35.5 & 8 & Soft & 0.082 & - & - & 91 & 70 \\
\hline 108 & Large & 0.5 & 4.2 & 10 & MQ & 0.098 & Off & Eq. (3) from C2016b & 27 & 70 \\
\hline 109 & Large & 0.5 & 4.2 & 0.1 & Soft & 0.098 & Off & Eq. (3) from C2016b & 26 & 70 \\
\hline 110 & Large & 0.5 & 4.2 & 0.1 & MQ & 0.098 & Off & Eq. (3) from C2016b & 28 & 70 \\
\hline 111 & Large & 0.005 & 4.2 & 10 & Soft & 0.098 & Off & Eq. (3) from C2016b & 26 & 70 \\
\hline 112 & Large & 0.005 & 4.2 & 10 & MQ & 0.098 & Off & Eq. (3) from C2016b & 27 & 70 \\
\hline 113 & Large & 0.005 & 4.2 & 0.1 & Soft & 0.098 & Off & Eq. (3) from C2016b & 28 & 70 \\
\hline 114 & Large & 0.005 & 4.2 & 0.1 & MQ & 0.098 & Off & Eq. (3) from C2016b & 28 & 70 \\
\hline 115 & Large & 0.5 & 76.5 & 10 & Soft & 0.066 & - & - & 387 & 70 \\
\hline 116 & Large & 0.5 & 76.5 & 10 & Soft & 0.098 & - & - & 6000 & 70 \\
\hline 117 & Large & 0.5 & 76.5 & 10 & MQ & 0.066 & - & - & 450 & 70 \\
\hline 118 & Large & 0.5 & 76.5 & 10 & $\mathrm{MQ}$ & 0.098 & - & - & 6060 & 70 \\
\hline 119 & Large & 0.5 & 76.5 & 0.1 & Soft & 0.066 & - & - & 455 & 70 \\
\hline 120 & Large & 0.5 & 76.5 & 0.1 & Soft & 0.098 & - & - & 6060 & 70 \\
\hline 121 & Large & 0.5 & 76.5 & 0.1 & MQ & 0.098 & - & - & 6060 & 70 \\
\hline 122 & Large & 0.016 & 76.5 & 10 & Soft & 0.066 & - & - & 455 & 70 \\
\hline
\end{tabular}




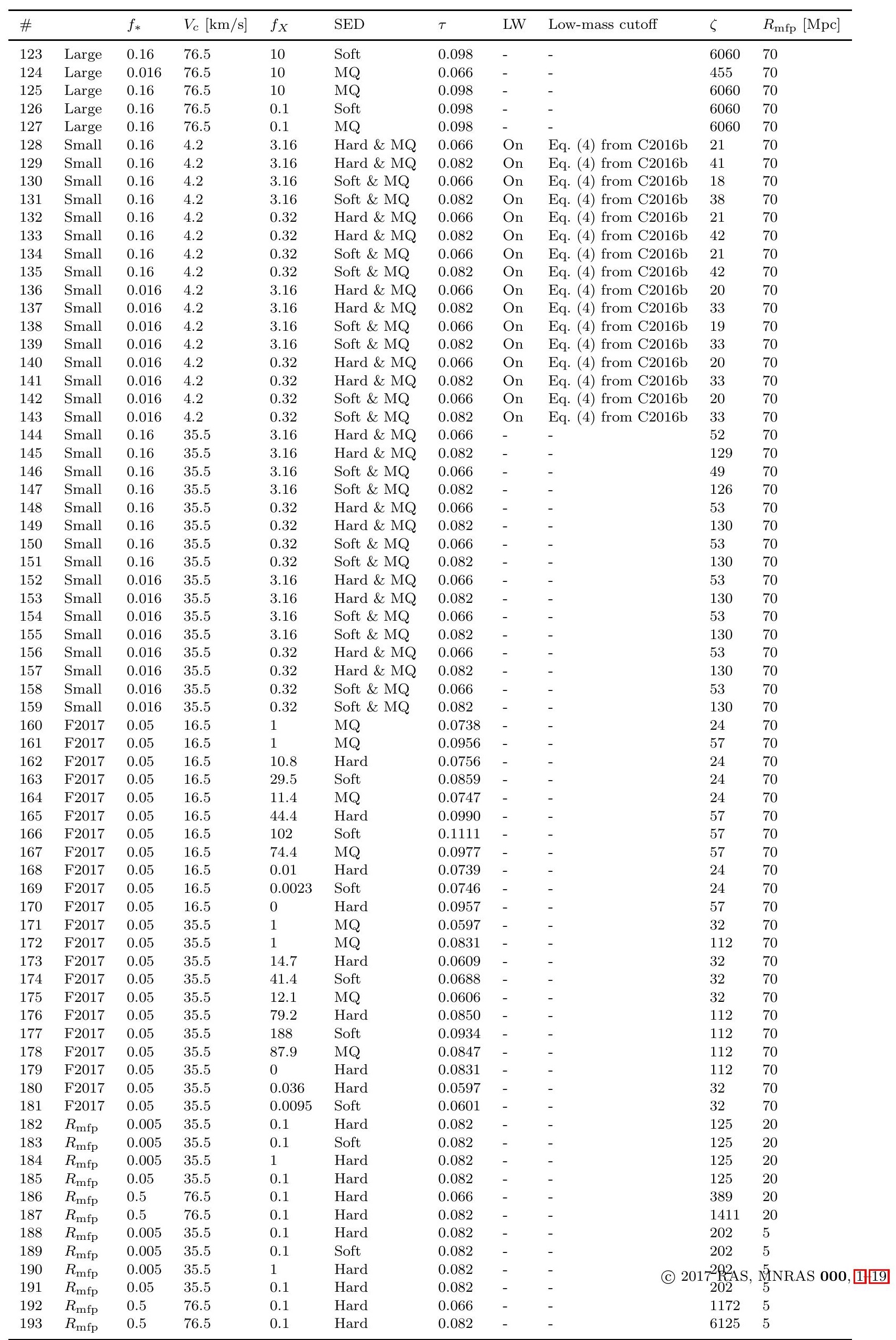


Strong X-rays
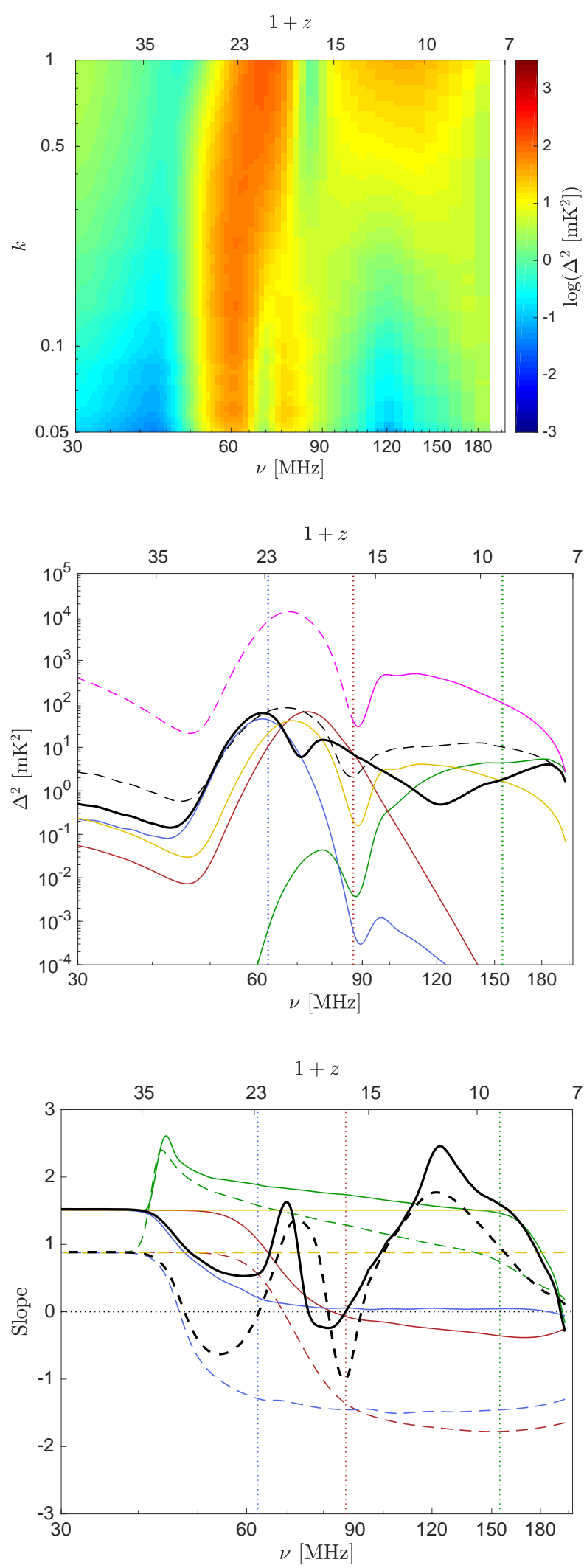

Massive halos
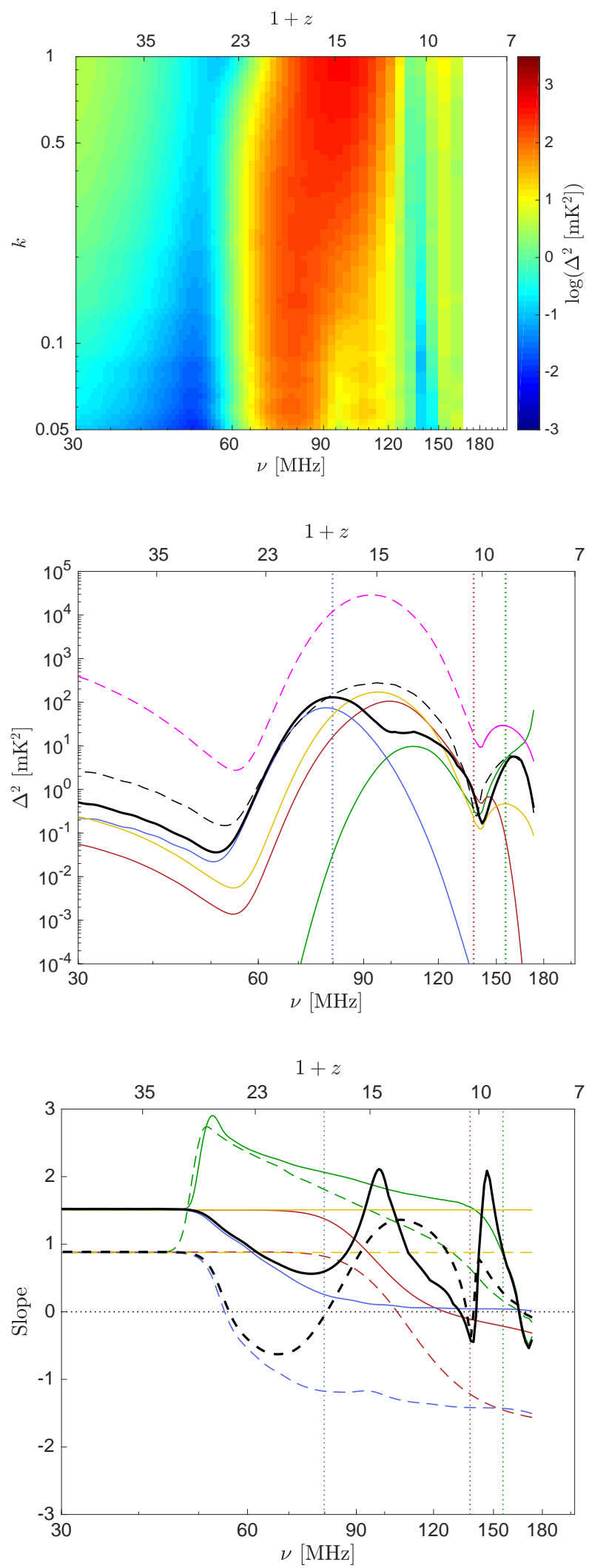

Figure B1. Variation of Figures 1 and 3 same line colors and styles. Left: The standard case except that $f_{X}=8$, model \#57. Right: The standard case except that $V_{c}=35.5 \mathrm{~km} \mathrm{~s}^{-1}$, model \#88. 
Low SFR
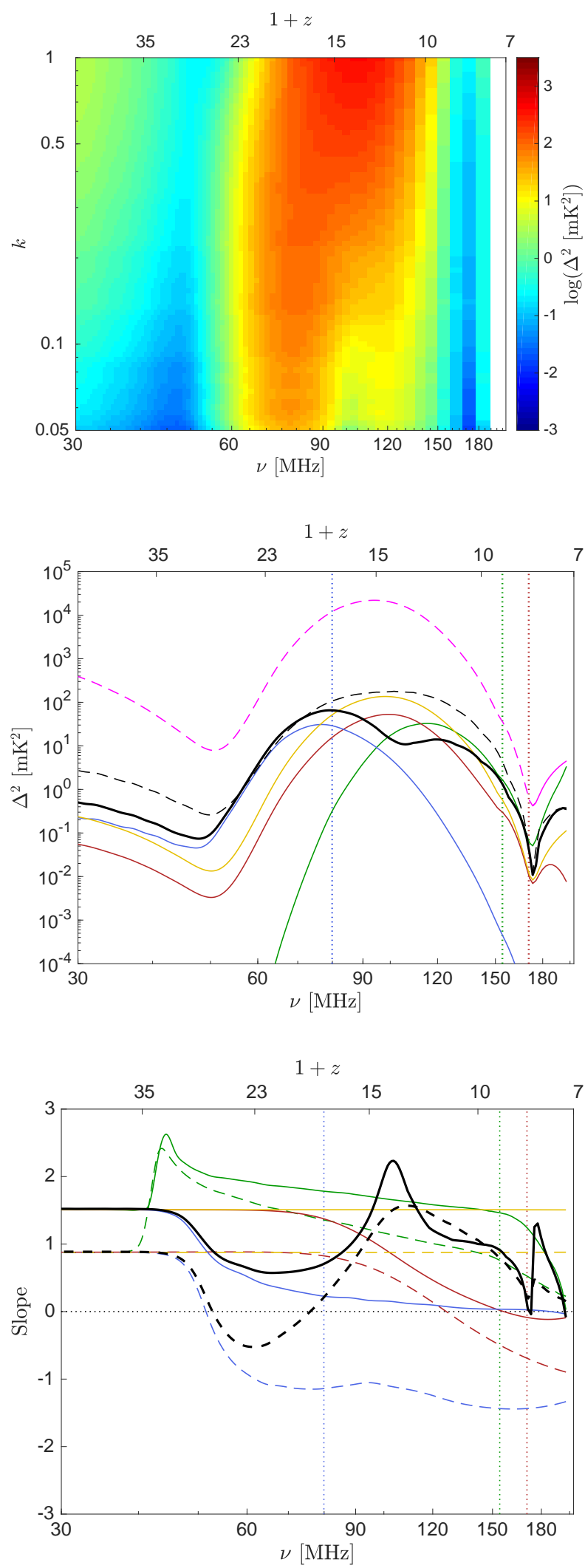

Very low mass halos
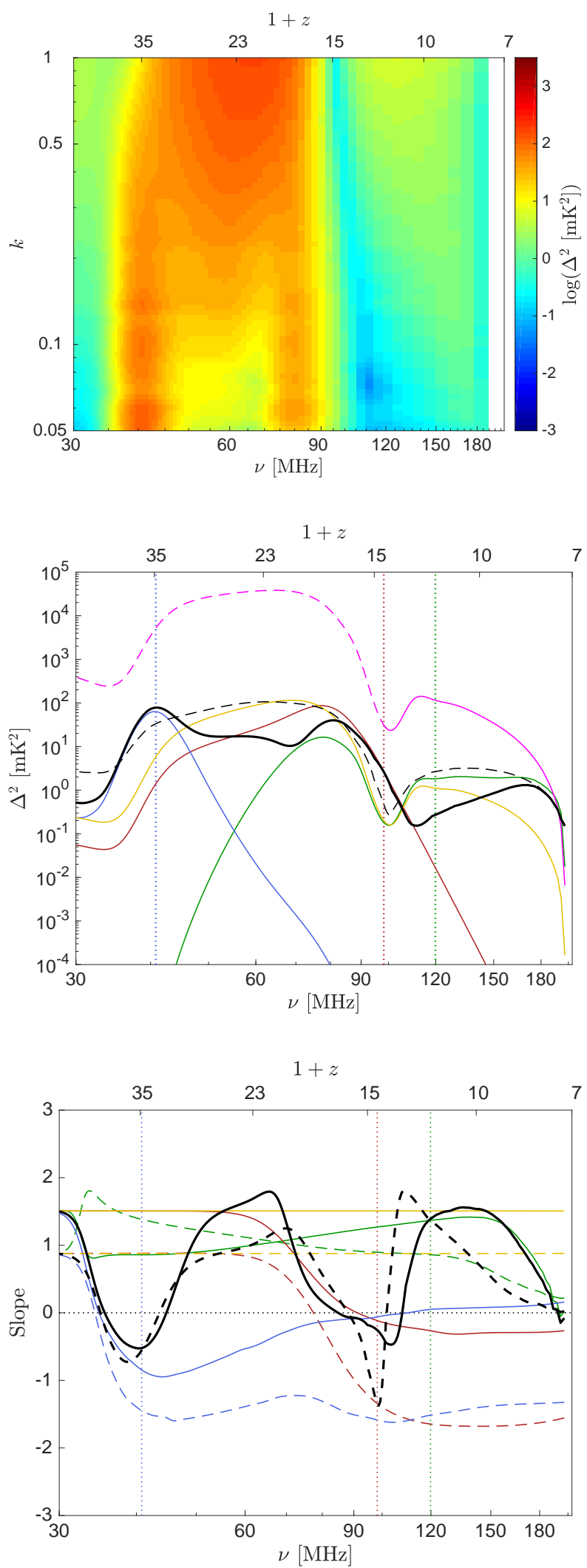

Figure B2. Variation of Figures 1 and 3 same line colors and styles. Left: The standard case except that $f_{*}=0.005$, model \#41. Right: High-redshift star formation dominated by very low mass halos, and X-rays produced only by miniquasars, model \#108. 


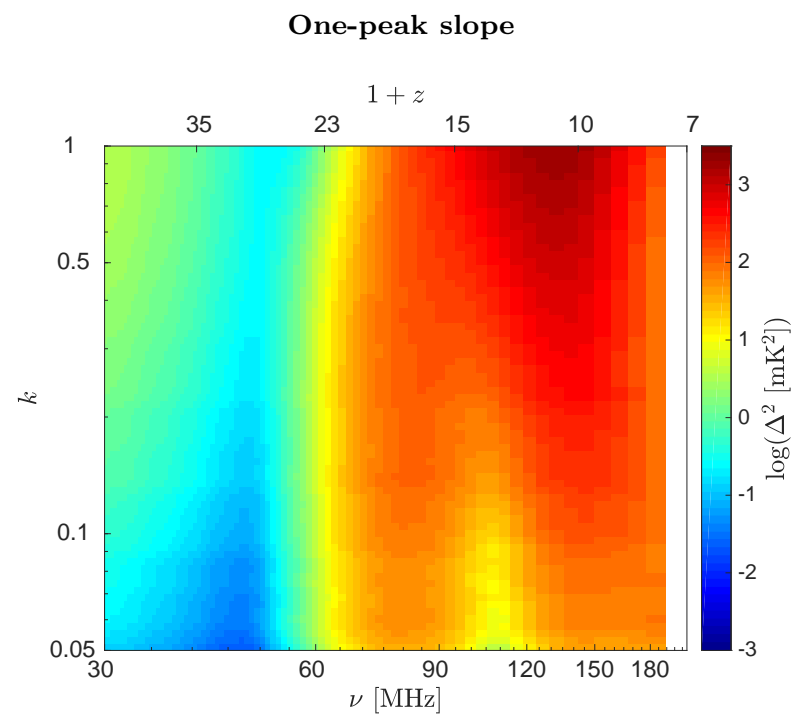

Slope with a bump
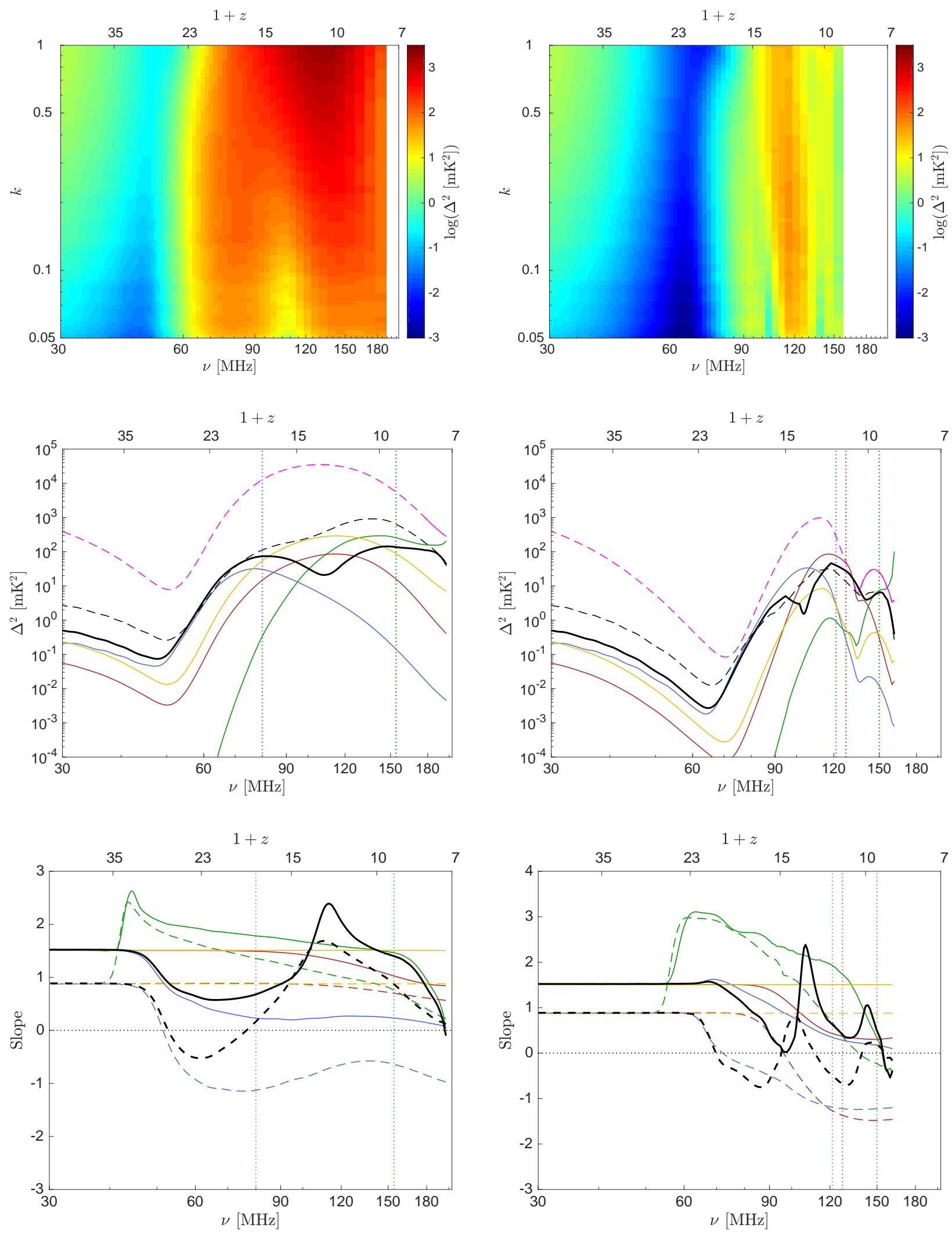

Figure B3. Variation of Figures 1 and 3 same line colors and styles. Left: A case with one minimum and one maximum in the slope, model \#37. Right: A case with an extra bump at very high redshifts due to late Ly $\alpha$ coupling, model \#122. 